\title{
La comprensión lectora inferencial de textos especializados y el rendimiento académico de los estudiantes universitarios del primer ciclo
}

\author{
Nelly Ugarriza Chávez*
}

El problema de la comprensión lectora inferencial de textos especializados en estudiantes universitarios de estudios generales de una universidad privada $(N=717)$ ha sido el eje principal de análisis en este estudio. La intención fue conocer las estrategias que utilizan para comprender un texto cientifico. Se han analizado las actividades cognitivas cuando leen un texto de psicología y mediante el análisis factorial se llegó a una solución de dos factores: F1, procesamiento macroestructural; y F2, modelo de la situación. Se recomienda la intervención de los docentes, quienes deben promover su acercamiento al texto y enseñarles estrategias adecuadas de comprensión de los textos cientificos de las asignaturas que tienen a su cargo.

Comprensión lectora inferencial / procesamiento macroestructural / análisis factorial

\section{Inferential comprehension of specialized texts and academic achievement of the students of the first courses at a private university}

The problem of inferential comprehension of specialized texts by students of general studies at a private university $(N=717)$ was the main axis of analysis in this study. The intention was to know the strategies that they used to understand a scientific text. When we analyzed by means of the factorial analysis the cognitive activities they undertook when reading a text of Psychology, we reached a solution of two factors: F1, macro structural processing; and F2 model of the situation. The intervention of the professors was recommended who must promote their approach to the text and teach them adequate strategies for the comprehension of scientific texts of the courses they are in charge.

Inferential comprehension / macro-structural processing / factorial analysis

* La autora agradece a Herlita del Castillo por el apoyo brindado.

Correo electrónico: nugarriz@yahoo.com 
Es de consenso casi general el sostener que la mayor parte de los ingresantes universitarios no poseen las habilidades lecto-comprensivas, ni las estrategias metacognitivas imprescindibles que los habiliten para el nivel superior, es decir se inician con una competencia lingüístico-discursiva muy poco desarrollada. Esta deficiencia significa un verdadero obstáculo para el aprendizaje del contenido de cualquier ciencia, por mínima que sea su complejidad. El nivel superior requiere lectores eficientes que puedan formular hipótesis, generar soluciones, comparar, analizar y describir hechos y procesos, clasificar, narrar, categorizar, reflexionar sobre los conocimientos adquiridos y los nuevos, todas estas son operaciones que se realizan desde la observación y la experiencia, pero en mayor medida desde la información que se lee.

Por otro lado, la mayoría de los estudios han indicado que el elevado número de deserciones académicas de estudiantes universitarios ocurre en el primer año (Cope, 1978; Tinto, 1975), y como lo señala Renner (1976), uno de los factores determinantes que contribuye al alto índice de estas bajas es la diferencia entre el nivel de enseñanza y el nivel de dominio de las destrezas de pensamiento de los alumnos.

La débil capacidad de la mayoría de los estudiantes para comprender lo que leen, criticar los textos, descubrir la estructura argumental, identificar los conceptos claves y las hipótesis que contiene un escrito, es la demostración del fracaso de toda una estructura de pensamiento que se fundamentó en la llamada educación bancaria y cuyos rezagos todavía están presentes. Los egresados de secundaria llegan a la educación superior con esquemas de pensamiento que privilegian la memoria por encima del pensar, ellos son expertos para acumular información, y "excelentes" en la repetición mecánica de datos y fórmulas; pero son incapaces de realizar raciocinios y formular hipótesis.

La presente investigación surge motivada por la preocupación manifiesta en los docentes universitarios al detectar diversas dificultades de los estudiantes en el manejo del idioma castellano, específicamente en lo referente a la comprensión lectora y a la redacción correcta y adecuada al nivel académico universitario. El problema se evidencia por la incapacidad del individuo para identificar las ideas principales expuestas en un texto, por la dificultad de captar la intención del autor y en la redacción incoherente de resúmenes de los textos objeto de lectura.

Se considera que son múltiples las variables que intervienen en el proceso de comprensión de textos científicos, y aunque a veces son tratadas independientemente interaccionan, por lo que su estudio es muy complejo. 
En el cuadro 1 se puede apreciar una clasificación de las variables en cuatro grandes componentes, referidos al profesor, al alumno, al texto y al contexto. En este estudio solo se destacarán los procesos cognitivos desplegados por el estudiante para la comprensión lectora de un texto especializado.

Como se sabe, la lectura de los textos involucra comprensión, interpretación e inferencia, lo que implica un proceso cognitivo muy complejo, que enfatiza el conocimiento de las estructuras lingüísticas, la cultura y el contexto. En la vida estudiantil es imposible concebir una actividad académica de aprendizaje sin la presencia de la lectura, la que constituye la clave para la formación profesional.

Leer es un proceso mental complejo, y quien lee debe concentrarse en el discurso del texto, al mismo tiempo que indaga, cuestiona y adopta una actitud crítica frente a él. El problema es que la mayor parte de nuestra lectura es acrítica, utilitaria, objetivista y solo la hacemos para informarnos de las generalidades del contenido del discurso. El reto es enfrentar el texto, comprenderlo, captando sus tesis y los mensajes que el escrito comunica.

En otras palabras, se lee para conocer la postura de un autor, para fundamentar o cuestionar una afirmación,

\section{Cuadro 1}

Variables que intervienen en el proceso de comprensión de textos

\begin{tabular}{ll}
\hline ComponenTES & Variables que influyen y regulan el proceso de comprensión \\
\hline Profesor & - Selección de contenidos del libro que se va a utilizar \\
& - Elección de las estrategias básicas de enseñanza \\
& - Sugerencias de estrategias de aprendizaje \\
& - Claridad y concisión en las instrucciones que se dan a los alumnos \\
& - Fomentar el interés de los alumnos para que lean textos científicos \\
Alumnos & - Conoción en las explicaciones que favorezcan a la comprensión \\
& - Hábitos de uso de textos \\
& - Elección de estrategias de aprendizaje \\
Texto & Interés en el tema del texto \\
& - Interés en reformular sus conocimientos \\
& - Lenganización (estructura) y estilo \\
& correcta \\
& - Inclusión de metáforas y analogías, inserción de figuras, fotografías, comenta- \\
& rios aclaratorios, explicaciones y elaboraciones con significación \\
Contexto & Condiciones implícitas en el ambiente de la clase, la interacción alumno-profe- \\
& sor, etcétera
\end{tabular}


para memorizar fórmulas o principios científicos, para conocer nuevos aportes al conocimiento de un objeto determinado, etcétera. Se escribe para organizar lo que se leyó, tomar notas de una clase, evidenciar lo que se sabe, presentar una ponencia, fundamentar una hipótesis, sistematizar datos, realizar un informe de investigación o presentar la tesis de licenciatura. Cada una de estas prácticas tiene su propia lógica o manera de organización, su propio código lingüístico y semántico, es decir, su propia práctica de lenguaje según se esté en el ámbito de las ciencias sociales, de la matemática, de la historia, de la biología, etcétera.

Nuestro propósito es destacar la comprensión de los textos que se leen en la universidad, que son los escritos de circulación académica que presentan alto grado de complejidad y de especificidad y cuyo manejo requiere operaciones intelectuales de mayor grado de abstracción.

Los textos presentan características particulares en cada área de conocimiento y requieren de estrategias $\mathrm{y}$ recursos específicos para su producción y comprensión. Gran parte de estos textos suponen un lector iniciado en la disciplina, con motivaciones específicas que comparte con los otros miembros de la comunidad científica y que debe esforzarse para situarse en el lugar del destinatario de ese escrito, reponiendo, construyendo o suponiendo lo que el autor ha dado por conocido.

\section{LA COMPRENSIÓN LECTORA}

La comprensión de textos es una actividad constructiva compleja de carácter estratégico, que implica la interacción entre las características del lector y del texto, dentro de un contexto determinado.

La construcción se elabora en base a la información que le propone el texto, pero esta se ve fuertemente enriquecida por las interpretaciones, inferencias e integraciones que el lector realiza con la intención de lograr una representación fiel y profunda de lo que el autor quiso comunicar, pero el lector en un momento dado puede ir más allá del mensaje comunicado por el texto. De allí que se puede afirmar que la construcción realizada por el lector tiene siempre un cierto tono especial de su persona, sea en los aspectos cognitivos, afectivos, actitudinales y volitivos, de manera que es imposible esperar que todos los lectores que leen un mismo texto puedan lograr una representación igual.

Así, el modo específico que asuma la interpretación dependerá de las interacciones complejas que ocurran entre las características del lector (sus intereses, actitudes, conocimientos previos, etcétera), del texto (las intenciones presentadas explícita o implícitamente por el autor) y el contexto (las demandas específicas, la situación social, etcétera) en que ocurra. Esto último hace que se considere al proceso 
de comprensión lectora una actividad esencialmente interactiva (De Vega, 1986).

Comprensión es entender el significado o contenido proposicional de los enunciados (oraciones) de un texto. Puede ser literal o inferencial. En la comprensión literal se accede estrictamente a la información contenida explícitamente en el texto: no se desbordan los contenidos proposicionales enunciados. Es decir, entender lo que el texto dice; en otras palabras, captar la información que presenta explícitamente siendo el primer paso hacia la comprensión inferencial y evaluativa o crítica. Dicha comprensión es necesaria tanto cuando se leen textos narrativos y poéticos, como cuando se leen textos informativos o expositivos. Cuando se examina este tipo de comprensión las preguntas se dirigen a reforzar si el lector comprendió: qué, quién, dónde, cuándo, con quién, cómo, para qué, etcétera, según lo que diga el texto. En cambio, en la comprensión inferencial -nivel superior de comprensión lectora- el pensamiento proposicional se apoya en la comprensión literal, pero la desborda. Este tipo de comprensión se refiere a la elaboración de ideas o elementos que no están expresados explícitamente en el texto. El alumno relaciona lo leído con sus propias situaciones. La información, implícita en la comprensión inferencial, se refiere a causas y consecuencias, semejanzas y diferencias, opinio- nes, hechos, conclusiones, mensajes inferidos sobre los personajes, el ambiente, y diferencias entre fantasía y realidad. Además, son importantes las preguntas inferenciales, que pueden estar basadas en el texto o en el lector: ¿qué piensan de...? ¿Cómo creen que...? ¿Por qué...? ¿Qué hubieran dicho o hecho ustedes?, etcétera.

La comprensión inferencial (González, 1996) puede reducirse a tres niveles jerarquizados: interpretación proposicional, reestructuración proposicional e implicación proposicional.

En la interpretación proposicional el lector apunta a la explicitación de contenidos subtextuales aludidos (Luria, 1979) por el enunciado, como sucede cuando se comprende un proverbio, un refrán, una metáfora o también cuando se traslada un problema enunciado verbalmente a una ecuación matemática.

En la reestructuración proposicional (Van Dijk, 1983) se reordenan los contenidos proposicionales a través de procesos de comparación, selección, eliminación, jerarquización y condensación proposicional que generan una macrocomposición, que es un breve resumen proposicional constituido por la idea central (macroestructura) y las ideas generales relevantes relacionadas con la macroestructura (macroproposiciones), que guían el procesamiento del texto total.

La interpretación proposicional apunta al procesamiento de oraciones y 
la reestructuración proposicional es condición de la comprensión profunda de un texto (comprensión inferencial).

En la implicación proposicional el lector establece consecuencias, efectos, derivaciones y relaciones causales no explícitas en el texto, pero que se desprenden de él.

\section{TEORÍA MACROESTRUCTURAL}

En torno al proceso de comprensión del discurso se han desarrollado gran número de trabajos empíricos desde hace aproximadamente tres décadas, que han permitido un avance considerable en la formulación de teorías sobre los mecanismos involucrados en dicho proceso, entre ellas se ha seleccionado la teoría macroestructural por su continuidad en el tiempo y adecuación a las observaciones empíricas y que aún se encuentra vigente.

La primera versión de la teoría (Kintsch \& Van Dijk, 1978; Van Dijk \& Kintsch, 1983) propugna que el lector establece dos niveles de representación del discurso: la microestructura y la macroestructura.

La microestructura es una representación semántica detallada del texto, por lo que también se denomina textobase. Es la estructura semántica lineal de un texto, al comprender un texto extraemos del mismo las distintas ideas que lo constituyen (proposiciones) y establecemos entre ellas una relación de coherencia lineal que presumiblemente será también semántica. La mi- croestructura corresponde al orden de conexión de las ideas básicas del texto, es decir provee las ideas elementales del texto (García, Elosúa, Gutiérrez, Luque \& Gárate, 1999).

La construcción de la microestructura ejecutada por el lector equivale psicológicamente a decir que el texto se considera legible, que puede leerse con una cierta fluidez y que posee una coherencia indispensable que le da sentido y lo hace inteligible (García, Elosúa, Gutiérrez, Luque \& Gárate, 1999).

Las actividades de microprocesamiento o microprocesos tienen que ver con la construcción de la microestructura, son de ejecución relativamente automática y son los subprocesos involucrados que se dirigen al establecimiento, codificación y coherencia local entre proposiciones. Los microprocesos involucrados en los niveles inferiores de la comprensión son los siguientes:

- El reconocimiento de las palabras escritas en los enunciados.

- La identificación o construcción de las proposiciones (ideas) a partir de los enunciados del texto.

- La vinculación de las proposiciones entre sí, encontrando el hilo conductor entre ellas: coherencia local entre proposiciones.

- Las inferencias, puentes necesarios para la vinculación e integración de proposiciones. 
La macroestructura constituye una representación sintética de lo más esencial de un texto, se procesa a partir de la microestructura y tiene carácter abstractivo; es decir supone una cierta reducción selectiva de la información, gracias a la cual se retiene el sentido del texto. Es algo así como la conjunción de las ideas (explícitas o implícitas) más importantes que se incluyen en el texto. Es la idea central de este, la que al ser identificada permite la comprensión textual. La macroestructura es el eje de la macrocomposición que se define como el conjunto de ideas principales del texto y que funciona como un modelo-resumen del texto. El proceso de elaboración de la macroestructura sigue tres etapas cíclicas fundamentales a partir de una entrada consistente en unidades proposicionales secuenciadas, que son sometidas a un complejo proceso de selección, eliminación, combinación y composición, para luego producir como salida una macroestructura textual.

Las macroestructuras textuales hacen referencia a los contenidos textuales, condensados o resumidos por el propio sujeto en macroproposiciones o paráfrasis abreviadas, pero que contienen la misma información. Formar una macroestructura de un texto es comprender el texto.

Las actividades de macroprocesamiento (macroprocesos) son aquellas que tienen que ver con la construcción de la macroestructura (representación semántica de naturaleza abstracta y global del texto) y del modelo de la situación derivada del texto (modelo mental análogo a las situaciones descritas en el texto) (véase García, Elosúa, Gutiérrez, Luque \& Gárate, 1999; Kintsch, 1998; MacNamara, Millar \& Bransford, 1991; Sánchez, 1993; Van Dijk \& Kintsch, 1983; Van Dijk, 1993-1994; Vidal-Abarca, 2000) (véase figura 1).

Los macroprocesos son de ejecución relativamente consciente, aunque el grado de conciencia en su aplicación también depende de factores tales como la complejidad del texto y el propósito del lector (véase Pressley, 1999). Los macroprocesos más relevantes son:

- La aplicación de las macrorreglas al tejido microestructural (dicha aplicación puede incluso ser recursiva): supresión o eliminación de las proposiciones irrelevantes, la generalización de las proposiciones con un cierto grado de solapamiento, y construcción o sustitución de ciertas proposiciones por otra información, no disponible en el texto, sino añadida por el propio sujeto (inferidas).

- La identificación de las macroproposiciones (jerarquización de las ideas del texto).

- La integración y construcción coherente del significado global del texto (coherencia global), a partir de las macroproposiciones. 
- La aplicación de inferencias basadas en el conocimiento previo.

- La construcción del modelo de la situación.

Un rasgo fundamental de este modelo es que tanto la microestructura como la macroestructura se representan en un formato proposicional. En consecuencia, el proceso de comprensión es la traducción desde un código fuente escrito en un lenguaje natural (el texto superficial) a un código interno o lenguaje mental (la representación proposicional).
La segunda versión de la teoría es la de construcción-integración (Kintsch, 1988), se aparta en diversos aspectos de la primera, pero mantiene algunos de sus rasgos fundamentales. Entre ellos, la concepción en dos etapas del proceso de comprensión y el formato estrictamente proposicional de las representaciones.

\section{Niveles de Representación}

Van Dijk \& Kintsch (1983) y Van Dijk (1983) propusieron tres niveles de representación del discurso y que son

Figura 1

\section{Componentes implicados en la lectura}

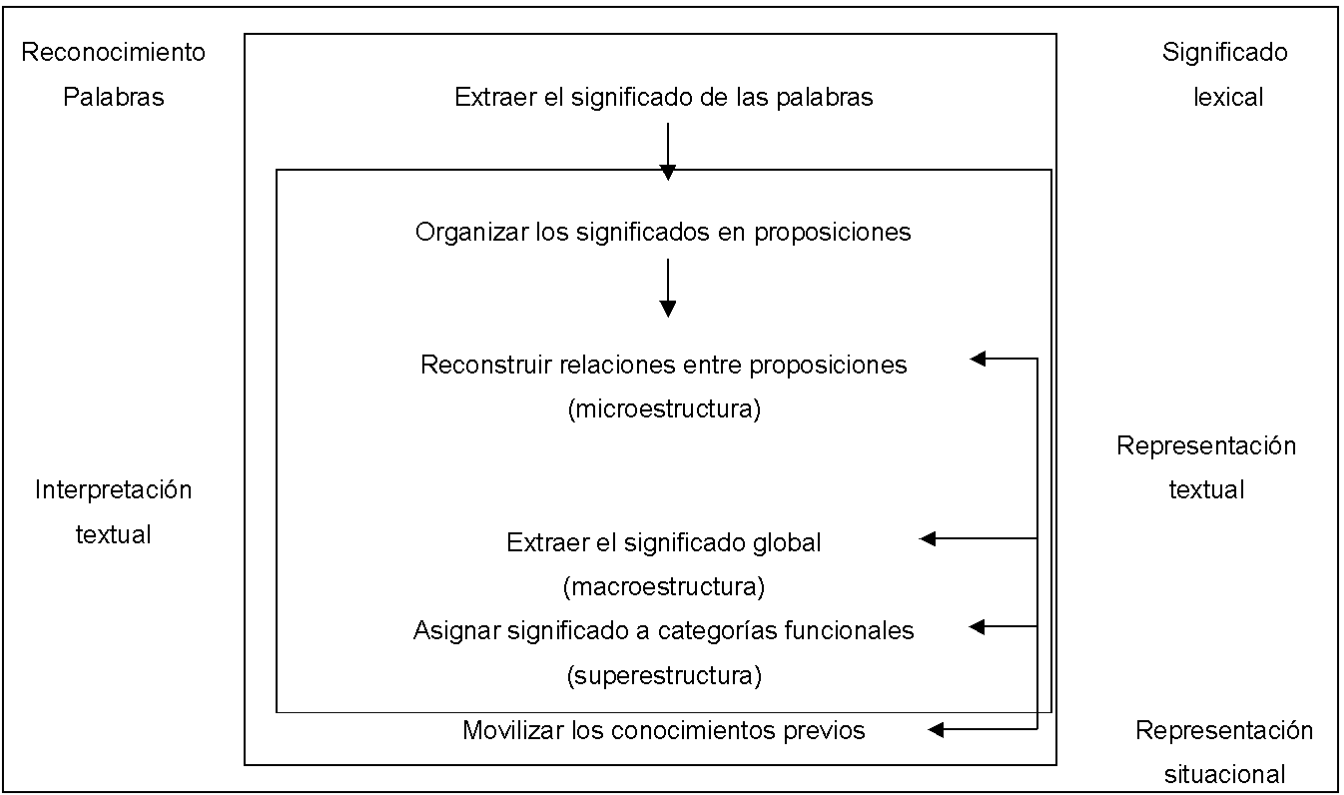

Tomado con algunas modificaciones de Orrantia \& Sánchez, 1994, en Díaz-Barriga \& Hernández (2002). 
necesarios para la comprensión eficiente.

En primer lugar, el lector elabora una representación de la forma superficial del texto, lo cual incluye la identificación de las palabras y el reconocimiento de las relaciones sintácticas y semánticas entre ellas.

En segundo lugar, genera el texto $b a$ $s e$, que representa el significado de las frases. El texto base se suele describir en un formato proposicional, que es básicamente adecuado para expresar el significado independientemente de la forma superficial del texto y se expresa mediante un lenguaje diferente al de las palabras y oraciones utilizado para la estructura superficial. El texto base es el conjunto interrelacionado de todas las proposiciones que representan el significado de un texto. Se trata de un conjunto que tiene estructura y no de una lista de proposiciones sin conexión entre sí.

$\mathrm{Y}$ en tercer lugar, Van Dijk y Kintsch propusieron los modelos de la situación que no son representaciones meramente lingüísticas o conceptuales de las frases, sino representaciones análogas a las que construimos en nuestra directa interacción con el mundo. Es la creación de un modelo mental, análogo al proporcionado por el texto, pero que se origina a partir de él, interviniendo en forma decisiva los conocimientos previos y las actividades inferenciales que el lector realiza. El modelo de la situación es el "mundo creado para el texto" y su logro consti- tuiría el nivel más complejo y quizá el más determinante para la comprensión de este.

El modelo de situación es la representación cognitiva de los acontecimientos, acciones, personas y, en general, de la situación sobre la que trata el texto (Maturano et al., 2002). Representar la situación que describe el texto supone incorporar gran cantidad de elementos no mencionados explícitamente en el texto, provenientes de la base de conocimientos del lector. La información procedente del texto se integra con dicho conocimiento, dando como resultado una representación más rica que la base del texto.

Los modelos de la situación se relacionan estrechamente con la comprensión. La creación de un modelo de la situación ha sido propuesto por varios autores como el criterio de qué es comprender un texto: un lector comprende un texto cuando es capaz de construir un modelo de la situación adecuado. Sin embargo, muchos textos son de difícil comprensión porque el lector, por falta de conocimientos, ni siquiera accede a los significados, es decir, no crea una base de texto. Pero conocer los significados de las palabras y frases de un texto no asegura crear un modelo de la situación apropiado. Este problema aparece, por ejemplo, cuando el autor usa términos muy generales. En este caso es muy difícil relacionar la información del texto con conocimientos relevantes que tiene el lector. 
Pero también procede identificando los aspectos y las claves organizativas o superestructurales que el autor del texto decidió seleccionar para comunicar sus ideas y gracias a ello puede construir el texto base. Esta habilidad para detectar las claves de organización superestructural ha sido denominada por algunos autores como conocimiento o sensibilidad a la estructura del discurso oral o escrito, y se desarrolla en la medida en que vamos interactuando con los diferentes géneros y estructuras textuales.

El conocimiento que el lector posee sobre las distintas superestructuras textuales puede contribuir significativamente a mejorar la comprensión (influye incluso en la construcción de la macroestructura) y en el recuerdo del texto.

\section{SUPERESTRUCTURAS DEL TEXTO}

Según la teoría de Kintsch y Van Dijk (1978), se conoce como patrón o estructura a la forma de organización de las ideas contenidas en el texto, y no tanto al contenido (el cual tiene que ver directamente con la microestructura y la macroestructura).

La superestructura textual (Van Dijk, 1983) es el tipo de texto. Son cinco las superestructuras o tipos textuales diferenciados (Bassols \& Torret, 1997): narrativos, descriptivos, argumentativos, explicativos e instructivos. Esta clasificación puede ser reducida a dos superestructuras textuales más generales: textos narrativos y expositivos.

A grandes rasgos, se suele distinguir entre textos narrativos y textos expositivos. Se trata de una distinción un tanto tosca pero útil, pues permite dividir el campo de estudio en dos grandes áreas que tienen una orientación y unas aplicaciones diferentes. Los textos expositivos son propios de la educación formal, su contenido es "impersonal" (ideas, argumentos, instrucciones, etcétera), inducen conocimiento en el lector; generalmente resultan difíciles y su lectura va acompañada de una cierta sensación de esfuerzo. Por el contrario, los textos narrativos están más próximos al discurso oral, su contenido es personal (colección de episodios con protagonistas, que tienen metas e intenciones, etcétera), inducen experiencia en el lector, son generalmente fáciles y nos divierten o entretienen. El estudio de la comprensión (y aprendizaje) de textos expositivos es un tema de interés educativo, ya que estos textos son herramientas para aprender. En cambio, el estudio de la comprensión de textos narrativos se dirige más bien a entender las características más universales de la comprensión. Es por esta razón que en el presente estudio se dedica una atención especial a la comprensión de textos expositivos.

Los textos expositivos son otro tipo de discurso, quizá más complejo por su grado de abstracción y arreglo lógico. 
Slater y Graves (1995) mencionan que no es fácil definir un texto expositivo; no obstante, proponen que este debe poseer las características siguientes:

- Tiene como función principal presentar al lector información de distinto tipo (teorías, predicciones, limitaciones, generalizaciones, conclusiones, personajes, fechas, etcétera).

- Provee de una buena cantidad de explicaciones y elaboraciones de la información provista.

- Son "directivos" en el sentido de proveer al lector de una guía basada en claves explícitas.

- Pueden incluir dentro de sí mismos elementos narrativos.

Este tipo de textos son continuamente utilizados en la prosa de distintas ciencias naturales (física, biología, entre otras), sociales (historia, geografía, etcétera) y otras disciplinas (por ejemplo, matemáticas, administración). Se dice que la superestructura expositiva predomina en los textos académicos, desde los ciclos finales de la educación básica hasta la educación universitaria. De hecho, los textos científicos son en esencia expositivos; aunque pueden asumir diferentes géneros (por ejemplo, el reporte científico, la monografía, el ensayo, etcétera).

Los textos expositivos usualmente contienen una serie de palabras claves o frases, utilizadas por el autor para organizar sus ideas. Es mediante el re- conocimiento de estas claves o frases que a los estudiantes se les facilita descifrar la estructura del texto.

Varios autores concuerdan en señalar que los textos expositivos asumen cinco tipos de superestructuras (Horowitz, 1985; Meyer, 1984; Richgels, McGee y Slaton, 1990; Sánchez, 1993; Slater y Graves, 1990; Gunning (1996), a saber: descripción, secuencia, comparación, causa y efectos y problema-solución.

Cada tipo de texto expositivo presenta un esquema (organizador figura textual) elaborado en tres niveles principales jerarquizados: nivel tópico, nivel de ideas principales (relación retórica) y nivel de información de detalles.

El nivel tópico, de mayor jerarquía, corresponde a la idea principal del texto; generalmente se coloca en la parte introductoria de modo explícito, o de forma sugerida o implícita.

El nivel de la relación retórica alude a la relación estructural que se da como argumentos lógicos en el discurso de las ideas principales, es decir, representa el tipo específico de relación estructural que predomina en él y que de algún modo lo caracteriza porque este varía en cada tipo de texto. Por lo general, la presentación de la relación retórica estructural y los marcadores de discurso que la apoyan constituyen la parte más sustancial del contenido y se encuentran en lo que podría llamarse el desarrollo del texto. 
El nivel jerárquico más bajo se refiere a la información de detalle que extiende la información retórica esencial del nivel inmediato superior. Dicha información amplía la relación retórica y se encuentra desarrollada a lo largo del texto.

Por último, un buen texto debe incluir una sección final de conclusión que cierra el texto. Sin embargo, no todos requieren de dicha sección, especialmente cuando se trata de textos expositivos breves. Cuando son largos o complejos sí es recomendable considerarla, bajo la forma de recapitulación o resolución.

Otra parte importante es el título, el cual debe ser claro, pertinente e informativo en el caso de textos expositivos educativos. Un buen título debe tener una relación esencial con el tópico del texto y con la información retórica superestructural.

A continuación se revisa cada tipo de texto expositivo:

- Texto descriptivo.- Es el tipo de superestructura que provee información acerca de un tema, concepto, evento, objeto, persona, idea, etcétera. Este patrón conecta ideas a través de descripciones, atendiendo las características importantes o los atributos del tema en cuestión. Es el patrón más común en los textos especializados. Los textos descriptivos son muy empleados en definiciones, biografías, cartas, narra- ciones y como partes importantes de textos informativos de diversa índole.

- Texto de secuencia.- En este tipo de superestructura las ideas se organizan por medio de un orden cronológico, por esta razón algunos autores lo denominan superestructura de orden temporal. Las palabras clave en los textos de secuencia son aquellas que expresan semánticamente un orden de eventos, sucesos o acciones en el tiempo.

- Texto comparativo.- En este patrón la organización del texto se realiza mediante la comparación de las diferencias y similitudes entre hechos, personas, conceptos, etcétera. Existen dos variedades: una es el llamado texto comparativo alternativo, donde simplemente se realiza la comparación entre dos cosas (temáticas, ideas o casos), dejando ver en qué y/o por qué son iguales o diferentes; en la otra modalidad, el texto comparativo adversativo, se tiene la firme intención de demostrar que una de las cuestiones comparadas es mejor o superior que la otra.

- Texto de causa-efecto.- La superestructura de este tipo de textos se fundamenta en una relación retórica del tipo causa-efecto (un evento o antecedente causa a otro evento o consecuente). Casi siempre se presenta la causa y una serie de explicaciones que la vinculan al efecto. También es posible que se presenten 
varias causas (en forma secuencial o en forma interactiva) y un solo efecto, o una sola causa y varios efectos.

- Texto de problema-solución.- Esta superestructura se articula en torno a la presentación de uno o varios problemas $\mathrm{y}$, posteriormente, al planteamiento de sus posibles soluciones. Los textos de problema-solución tienen un componente secuencial y/o causal.

La investigación realizada sobre la comprensión y el recuerdo de textos narrativos y expositivos ha demostrado los siguientes hallazgos relevantes:

Se han podido identificar algunas diferencias esenciales entre las características intrínsecas de los textos narrativos y los expositivos. Algunas de las diferencias más relevantes son las siguientes:

- Se acepta que los textos narrativos tienen una gramática mucho más regular que los textos expositivos.

- Los textos narrativos se fundamentan esencialmente en relaciones de coherencia causal y, sobre todo, motivacional (los personajes realizan planes y acciones para el logro de metas), mientras que los textos expositivos emplean frecuentemente relaciones de propiedad (relaciones descriptivas cuya función consiste en vincular entre sí los enunciados sobre un objeto, cómo se relaciona con otras cosas y de qué se compone) y de apoyo (relaciones argumentales que conectan los enunciados que contienen afirmaciones generales con otros enunciados que apoyan o contradicen la veracidad de tales afirmaciones).

- La estructura de los textos narrativos es muy regular y constante de un ejemplar a otro, mientras que en los textos expositivos esto no ocurre así.

- Los textos narrativos están más asociados con nuestras experiencias cotidianas y se leen con mayor frecuencia que los textos expositivos.

En general, se acepta que existe una mayor dificultad para la comprensión y recuerdo de textos expositivos, en comparación con los textos narrativos. Se ha demostrado en varios trabajos que:

- Los textos expositivos exigen más esfuerzo cognitivo (por ejemplo, más procesos atencionales, mayor tiempo de lectura en las frases) y despiertan menos interés que los textos narrativos.

- La elaboración de inferencia resulta mucho más probable en la lectura de textos narrativos que en la de textos expositivos; de hecho, algunos autores sostienen que la generación de inferencias en los textos narrativos es más automática, mientras que en los textos positivos tiende a ser más reflexiva y controlada. 
Los niños están más familiarizados con los discursos narrativos desde temprana edad. Todos los cuentos populares, los cuentos de hadas y leyendas (presentados primero en forma oral y posteriormente por vía escrita), e incluso las historietas a las que se enfrentan por medio de la televisión o los cómics (con modalidad oral, visual y/o escrita), siguen un patrón estructural similar, aunque con ciertas variantes. Por tanto, la comprensión y el recuerdo de dicho tipo de textos desde la niñez suelen ser mejores en comparación con otros.

No existe un acuerdo total sobre las diferencias debidas a la edad para comprender los cinco tipos de textos expositivos. A partir de los años correspondientes a los últimos grados de la educación básica, parece que se va adquiriendo la sensibilidad necesaria para identificar las superestructuras expositivas en los textos (primero en los textos descriptivos de colección y enumerativos y después en los de covariación, causaefecto, comparativo-adversativos, y problema-solución). De hecho, la tendencia de los lectores más pequeños, quienes no demuestran mucha sensibilidad a las superestructuras expositivas, es recordar los textos expositivos (por ejemplo, un texto causal o de problemasolución) como un mero listado de ideas, lo cual afecta seriamente la comprensión de las relaciones entre ideas.

Como ya se ha indicado, son las diferencias individuales las que se tornan más claras; es decir, a partir de la niñez tardía y durante toda la adolescencia, los buenos lectores comienzan a distinguirse por la sensibilidad para identificar y hacer uso de las relaciones retóricas (identificando las relaciones de primer orden y utilizando los marcadores sintácticos), primero en el campo de la comprensión de textos y un poco más tardíamente en el campo de la composición escrita. Los malos lectores nuevamente tienen dificultades para identificar las superestructuras -especialmente las más complejas- cuando leen y tienden a utilizar la estrategia de listado en su recuerdo.

La conciencia sobre la estructura de los textos expositivos con relación a la de los textos narrativos se desarrolla tardíamente en los estudiantes, ya que estos textos requieren un nivel de pensamiento abstracto más elevado que el de los textos narrativos. Es por eso que la utilización de textos expositivos está monitoreada constantemente por los maestros en los niveles primarios y no es hasta los grados más altos de la escuela elemental o quizás de la escuela intermedia que los estudiantes trabajan estos textos de manera independiente. Es en este nivel que se debe estimular que el estudiante tome conciencia sobre cómo los textos expositivos o informativos están estructurados, para que él pueda utilizar el reconocimiento tanto de la estructura externa como de la estructura interna, de un determinado texto, en provecho de su comprensión lectora. De manera que si se desa- 
rrolla un esquema sobre la estructuración con la cual el autor presenta sus ideas en un texto el estudiante podrá leer, usar y comprender mejor un texto (Irvin, 1998).

En nuestro medio son escasos los estudios reconocidos sobre la comprensión lectora y el rendimiento académico de los estudiantes universitarios peruanos, encontrándose los trabajos de González como aportes básicos y relevantes para los propósitos de la presente investigación. En el 2001 publicó un estudio sobre el papel del componente superestructural en la elaboración de las macroestructuras. Exploró una muestra de 62 estudiantes de una universidad privada, compuesta por 48 mujeres y por 14 varones. Halló que el tipo de texto que presenta mayor dificultad es el expositivo, seguido del argumentativo y narrativo.

En el 2000, González analizó los factores que influyen en el desempeño matemático de estudiantes universitarios iniciales. Exploró una población de 67 estudiantes de los ciclos iniciales de Estudios Generales de una universidad privada. Los dividió en dos grupos: 27 de alto rendimiento y 40 de bajo rendimiento. El perfil de dificultades común a ambos grupos de alto y bajo rendimiento es el siguiente: debilidad en los esquemas operatorios lógicoformales de probabilidad, distribución normal y combinaciones en el área que denomina operatoria. En cálculo, el problema común son las estimaciones, este aspecto del pensamiento matemático está vinculado a los procesos metacognitivos. En el área de problemas, en ambos grupos se presenta mucha debilidad en identificar lo relevante de un enunciado narrativo desde la perspectiva del planteamiento de un problema matemático; es un proceso vinculado a la comprensión más profunda de un texto: destacar la idea principal.

En 1998, realizó un estudio sobre la comprensión lectora en estudiantes universitarios iniciales, participaron 41 sujetos, para ser contrastados con una muestra similar aleatorizada que fueron extraídos de la muestra original de 311 de una universidad privada. Las categorías de competencia lectora hallados mostraron la prevalencia de lectores dependientes en los textos básicos informativos, documentarios y numéricos y de lectores deficitarios en los textos literarios, humanísticos $\mathrm{y}$ científicos.

En 1997, González y Quesada exploraron la comprensión lectora en dos grupos de estudiantes secundarios y universitarios $(\mathrm{N}=103)$, para conocer sus niveles de analfabetismo funcional. Se aplicó la técnica cloze a lecturas básicas de alfabetización informacional (en prosa), documentaria y numérica (cálculo); y a lecturas complementarias de dominio alfabético: textos literarios, científicos (divulgación) y humorísti$\cos$ (ensayo). 
Los promedios de los estudiantes secundarios se encontraban por debajo del nivel crítico, revelando analfabetismo funcional. Los estudiantes universitarios superan en promedio por un punto debajo de ese límite en cuanto a lo que respecta a los textos básicos, pero también están por debajo de ese límite en cuanto a los textos complementarios. El principal instrumento de trabajo académico es el lenguaje escrito y los estudiantes evaluados exhiben un decepcionante rendimiento lector.

Es importante señalar que la comprensión de la lectura en el nivel de la superestructura quizás no sea lo suficientemente explorada mediante la utilización de técnicas cloze o técnicas de neologismos, y que estas a su vez no sean las únicas formas de explorar la comprensión textual, de allí que en la presente propuesta se diseñará una prueba de comprensión lectora "convencional" basada en un texto especializado de psicología, que explore la comprensión inferencial considerando el tipo de texto expositivo.

A partir de las consideraciones anteriores el presente estudio está dirigido a resolver las siguientes interrogantes:

- ¿Cuál es el nivel de rendimiento en el texto convencional de psicología del estudiante de los ciclos iniciales de las diferentes carreras, cuando se analizan las actividades propuestas: realizar un resumen, poner un título, extraer la idea principal, interpretar gráficamente lo que le sugiere el texto y responder a tres interrogantes sobre este?

- ¿Varía la comprensión lectora inferencial cuando se considera la edad, el sexo, la carrera, las veces que el alumno cursó la asignatura de Psicología y el nivel de rendimiento en esta?

- ¿Qué relación existe entre la comprensión lectora inferencial y la estrategia elegida que más le ayudó al estudiante a comprender el texto?

- ¿Qué asociación existe entre la comprensión lectora inferencial, la autopercepción de sus logros escolares y el rendimiento en la asignatura de Psicología de estudiantes universitarios del ciclo inicial?

\section{Objetivos}

- Determinar la confiabilidad mediante el coeficiente Alfa de Cronbach de las pruebas de comprensión lectora inferencial (CLIU) y de la prueba de neologismos González-Ugarriza-Bazán (GUB).

- Determinar la validez de constructo de la prueba comprensión lectora inferencial (CLI-U), mediante la técnica del análisis factorial y la correlación entre las pruebas GUB y CLI-U. 
- Describir los rendimientos en comprensión lectora inferencial con base en las actividades solicitadas sobre el texto: realizar un resumen del texto de psicología, poner un título, extraer la idea principal, interpretar gráficamente lo que le sugiere y responder a tres interrogantes sobre este.

- Describir y comparar los rendimientos considerando el sexo, la edad, la carrera, las veces que el estudiante cursó la asignatura y el nivel de rendimiento en esta.

- Describir la relación entre comprensión lectora inferencial y la estrategia elegida que le ayudó a comprender el texto.

- Analizar la relación entre la autopercepción del rendimiento en las asignaturas de secundaria y la comprensión lectora inferencial.

- Describir la relación entre el rendimiento en la asignatura de Psicología y la comprensión lectora inferencial.

\section{Método}

La investigación tiene un carácter descriptivo, asumiéndose como variables independientes: la edad, el sexo, la carrera, la repitencia en la asignatura de Psicología y el nivel de rendimiento en esta y como variable dependiente la comprensión lectora inferencial de los estudiantes del primer ciclo. Además, es un estudio correlacional, porque analiza la asociación entre las variables: estrategia que más ayudó al estudiante a comprender el texto, autopercepción de los logros escolares y rendimiento en el curso de Psicología.

\section{Población y muestra}

La población considerada está conformada por 2.673 estudiantes matriculados en los ciclos iniciales del año 2005 de una universidad particular de la ciudad de Lima, habiéndose encuestado una muestra de 717 alumnos, que representan el 27 por ciento de la población. La muestra ha sido estratificada según las variables consideradas en el problema de investigación.

Se solicitó previamente el permiso de las autoridades del Programa de Estudios Generales de la Universidad y se trató de aplicar el máximo de cuestionarios posibles, eligiendo para ello los horarios de la teoría del curso de Psicología, de modo que pudieran estar el mayor número de alumnos matriculados. La duración de la aplicación de las pruebas fue de aproximadamente 50 minutos.

En la tabla 1 se observa que el 50,8 por ciento de estudiantes tiene más de 18 años, el 54,1 por ciento es de sexo masculino. La escuela más representada es la de Humanidades (48,4\%), seguida por Ingeniería $(30,1 \%)$ y luego la de Negocios $(21,5 \%)$. 
Tabla 1

Distribución de la muestra de alumnos matriculados en los dos primeros ciclos de estudios generales de una universidad particular $(N=717)$

\begin{tabular}{llll}
\hline Variables & & $\mathrm{f}$ & $\%$ \\
\hline \multirow{2}{*}{ Edad } & & & \\
& 16 a 17 años & 353 & 49,2 \\
Sexo & 18 años y más & 364 & 50,8 \\
& & & \\
& Masculino & 388 & 54,1 \\
Carrera & Femenino & 329 & 45,9 \\
& & & \\
& Humanidades & 347 & 48,4 \\
Veces que cursó la carrera & Negocios & 154 & 21,5 \\
& Ingeniería & 216 & 30,1 \\
& Primera vez & 387 & 54,0 \\
& Pasaron el curso & 254 & 35,4 \\
& Repitentes & 76 & 10,6 \\
\hline
\end{tabular}

En cuanto al rendimiento en el curso de Psicología, se han conformado tres grupos contrastantes de acuerdo al criterio de rendimiento académico en la asignatura de Psicología. La clasificación de los grupos es la siguiente:

- Grupo 1: Alumnos del primer ciclo 2005-2, que recién iniciaban la asignatura de Psicología.

- Grupo 2: Alumnos de rendimiento satisfactorio en la asignatura de Psicología del primer ciclo 2005-1 (calificativo por encima de 14).

- Grupo 3: Alumnos de rendimiento insatisfactorio del primer ciclo 2005-1 (calificativo por debajo de 11). Que hayan reprobado una o dos veces (repitentes).
En la tabla 1 se aprecia que el 54\% cursaban por primera vez la asignatura de Psicología, el 35,4\% habían aprobado la materia y el 10,6\% eran repitentes. Todos ellos se encontraban matriculados en el semestre 2005-2.

\section{TÉCNICAS DE RECOLECCIÓN DE DATOS}

Evaluación de la comprensión lectora: cuestionarios

Se utiliza la técnica de neologismos para medir el rendimiento inferencial en los tres tipos de superestructura textual: narrativos, argumentativos y expositivos. Cada texto está compuesto por seis oraciones, que enuncian algún significado de un mismo neologismo y la tarea consiste en identificar el significado del neologismo. 
La autora del presente proyecto ha realizado un análisis psicométrico del cuestionario propuesto por González (2001) en una muestra de alumnos de una universidad particular $(\mathrm{N}=490)$ de diferentes carreras de letras y ciencias. Se procedió a realizar un análisis más depurado de la prueba de comprensión textual utilizando los programas estadísticos SPSS 12.0 y el ITEMAN, lo cual dio lugar a una nueva versión de este instrumento, que se denominó prueba de comprensión textual González, Ugarriza y Bazán (GUB) (Ugarriza, 2004). De 30 ítems, 10 fueron eliminados por este procedimiento y así se utilizó la prueba para el análisis de datos, mejorando su validez, índice de discriminación y nivel de dificultad.

\section{Prueba de comprensión lectora "convencional"}

Para el presente estudio se ha elaborado una prueba de comprensión lectora inferencial basándose en el contenido del texto de psicología que llevan los estudiantes del primer ciclo. Su confiabilidad y validez se ha determinado con la muestra investigada y los datos se presentan en los resultados.

Para detectar los inconvenientes de lecto-comprensión con que los estudiantes ingresan a la Universidad se han seleccionado seis actividades, que pueden surgir en el proceso de comprensión inferencial de un texto escrito especializado de psicología.
Para realizar la experiencia piloto se trabajó con el grupo de alumnos ingresantes a una universidad particular que no hayan llevado la asignatura de Psicología durante el primer semestre del 2005. Se plantearon cuatro textos:

- "Edad, inteligencia, sabiduría y creatividad, ¿cambio o estabilidad a lo largo de la vida?"

- "Cómo cambiar el trabajo en juego: La motivación intrínseca en el salón de clases".

- "La teoría del yo de Rogers: Convertirse en una persona plena".

- "La violencia juvenil".

El trabajo consistió en el análisis de diversos textos de tipo expositivo, cuyos contenidos se relacionaban con temas científicos extraídos de textos de psicología.

En la elección del texto se tuvo en cuenta que el contenido desarrollado sea una exposición lógica (buena organización de la información; buen tratamiento lingüístico) y psicológicamente significativos (que plantee una distancia cognitiva óptima para los conocimientos previos) para los alumnos, y que sea pertinente a los aprendizajes que se quieren promover en ellos.

\section{DISEÑO DE LA PRUEBA}

La prueba ha consistido en efectuar, a partir de la lectura del texto, las siguientes actividades: 
- realizar un resumen;

- extraer la idea principal;

- poner un título;

- interpretar gráficamente lo que le sugiere el texto; $y$,

- responder tres preguntas sobre el texto.

Además, a modo de autoanálisis de las estrategias de comprensión utilizadas por cada alumno, se les solicitó que entre las opciones anteriores señalen las que fueron más útiles para su comprensión.

La elaboración de resúmenes, como estrategia elaborada por el alumno, ha sido investigada en varios estudios realizados a partir del modelo macroestructural de Van Dijk y Kintsch (1983). De acuerdo con estos autores la construcción de la macroestructura (jerarquización de la información relevante y reducción de la información irrelevante) tienen que ver directamente con los resúmenes, se elabora a partir de la aplicación de las macrorreglas y de la superestructura.

El resumen constituye, sin duda, una poderosa estrategia, porque quien lo elabora se obliga a profundizar y reflexionar de manera consciente sobre la macroestructura y la superestructura del texto, sobre el modelo de la situación y a reformular con los códigos y el vocabulario personal aquello que el autor nos quiso decir por medio del texto. Si bien el resumen es esencialmente reproducti- vo (porque se parte de un texto al que se quiere reducir a sus ideas más relevantes), también es una actividad compleja y reconstructivo-creativa.

La idea principal, siendo también parte de la macroestructura, se refiere a la identificación o construcción del enunciado o enunciados de mayor relevancia que el autor utiliza o sugiere para explicar el tema. La idea principal se podría construir contestando a la pregunta: ¿cuál es la idea más importante que el autor utiliza (o sugiere) para explicar el tema? Primero se requiere especificar el tema, que es aquella parte de la macroestructura que nos permite responder a la pregunta $¿ \mathrm{de}$ qué trató el texto?, y se expresa por lo menos mediante un enunciado simple, el cual puede coincidir con el título o con el subtítulo del texto.

La identificación de la idea principal involucra las siguientes actividades: a) construir una representación global del texto (implica el tema); b) hacer juicios sobre la importancia de la información y reducir la información trivial, secundaria y redundante, y c) consolidar la idea principal, después de hacer un análisis reflexivo sobre el peso de aquellas ideas que se consideraron relevantes. En ocasiones, la idea importante se encuentra de forma explícita en el texto (lo cual requiere realizar las anteriores acciones hasta encontrarla); sin embargo, en otras situaciones la idea no aparece explícitamente en el texto y es necesario construirla. 
Respecto al título, Mayer (1986) destaca su importancia para lograr una mejor comprensión del texto. Se ha considerado que, al solicitar a los alumnos esta actividad junto con la búsqueda de la idea principal, puede revelar el grado de comprensión alcanzado. Para realizar esta actividad, el alumno debe interrelacionar globalmente las ideas del texto y determinar de qué trata este.

La interpretación gráfica también se ha considerado como una actividad muy importante para la comprensión del contenido del discurso, ya que los alumnos realizan una representación visual del texto: un organizador gráfico, una tabla causa-consecuencia o comparativa, u otros esquemas conceptuales.

El uso de los cuestionarios formulados sobre un texto puede convertirse en una eficaz herramienta de aprendizaje y de evaluación en el proceso de estudio de un tema.

La prueba se presenta en el anexo. Cabe señalar que se les permitió tener el texto durante el desarrollo de la prueba para evitar la memorización de este.

Respecto a la evaluación de las respuestas a las preguntas sobre el texto para calificar los resultados se ha adoptado una escala tricotómica: bueno, regular o malo. Los criterios adoptados son descritos al analizar cada actividad.

\section{Tipo de preguntas}

Respecto al nivel de procesamiento que demanda la pregunta no se han ele- gido reactivos de alto nivel de estructuración: "falso-verdadero", "correspondencia" y "complementación", de "respuesta breve" o "completamiento" y los de "opción múltiple" porque son factuales o reproductivas, que inducen un procesamiento superficial de la información, en tanto que solicitan al lector el recuerdo literal y de detalles sobre la información, y más bien se ha preferido preguntas de "respuesta abierta" y de "desarrollo de temas", que a diferencia de los anteriores demandan actividades de mayor complejidad y procesamiento, tales como comprensión, elaboración conceptual, capacidad de integración, creatividad, habilidades comunicativas, capacidad de análisis y establecimiento de juicios reflexivos o críticos. Es decir, son preguntas implícitas y constructivas que promueven el procesamiento profundo porque demandan la comprensión inferencial, la aplicación y la integración de la información.

\section{Cuestionario de datos generales}

A través de un cuestionario se indagó los datos sobre las variables demográficas, la autopercepción del alumno acerca de su rendimiento en secundaria en los cursos de Lengua y Literatura, Matemáticas y Ciencias, considerándose los siguientes calificativos: muy bueno, bueno, regular y malo. Además, se les preguntó las veces que habían llevado el curso de Psicología. 
Actas del curso de Psicología

Se registró solo la nota final del curso de Psicología del primer semestre académico 2005, que fue reportada por Estudios Generales.

\section{Resultados}

Análisis psicométrico de los instrumentos de recolección de datos

En primer término se procederá a realizar un análisis psicométrico de los instrumentos de recolección de datos, determinando la confiabilidad y la validez de las pruebas de comprensión lectora inferencial (CLI-U) y la prueba de comprensión lectora GUB.

Para determinar la consistencia interna de las pruebas mencionadas se utilizó el estadístico Alfa de Cronbach. En la tabla 2 se presentan los resultados para la prueba CLI-U, examinándose un coeficiente correlación ítem-total corregida que exceden al criterio mínimo esperado de .20. El Alfa para la prueba completa es de .5676 , siendo este valor moderado, esto se atribuye a que son solo siete los ítems evaluados.

Cuando se examina la confiabilidad de la prueba GUB, el coeficiente Alfa observado fue de .7581 , el que resulta aceptable, cuando se examina la correlación ítem-total corregida, los elementos $4 \mathrm{~N}$ y $11 \mathrm{~A}$ arrojan valores por debajo de .20, pero su eliminación no incrementa sustancialmente la consistencia de la prueba (tabla 3 ).
Tabla 2

Confiabilidad de la prueba de comprensión lectora inferencial en estudiantes universitarios (CLI-U) $(\mathrm{N}=717)$

\begin{tabular}{lcc}
\hline Ítems & $\begin{array}{c}\text { Correlación } \\
\text { item-total } \\
\text { corregida }\end{array}$ & $\begin{array}{c}\text { Alfa si el } \\
\text { ítem } \\
\text { es eliminado }\end{array}$ \\
\hline Resumen & .2858 & .5310 \\
Idea principal & .3186 & .5225 \\
Título & .2794 & .5372 \\
Figura & .2717 & .5389 \\
Conc. Motivac. & .2365 & .5485 \\
Exp. Vi-Vd & .3132 & .5209 \\
Aplicaciones & .3526 & .5046 \\
\hline
\end{tabular}

Alfa $=.5676$

Tabla 3

Confiabilidad de la prueba de comprensión lectora inferencial mediante la técnica de neologismos en estudiantes universitarios (GUB) $(N=717)$

\begin{tabular}{lcc}
\hline Ítems & $\begin{array}{c}\text { Correlación ítem-total } \\
\text { corregida }\end{array}$ & $\begin{array}{c}\text { Alfa si el ítem } \\
\text { es eliminado }\end{array}$ \\
\hline $1 \mathrm{~A}$ & .2871 & .7509 \\
$2 \mathrm{E}$ & .3404 & .7470 \\
$3 \mathrm{~A}$ & .3199 & .7487 \\
$4 \mathrm{~N}$ & .1587 & .7607 \\
$5 \mathrm{~A}$ & .2037 & .7555 \\
$6 \mathrm{E}$ & .3896 & .7431 \\
$7 \mathrm{~A}$ & .3789 & .7457 \\
$8 \mathrm{E}$ & .3636 & .7451 \\
$9 \mathrm{~N}$ & .2710 & .7522 \\
$10 \mathrm{E}$ & .1964 & .7560 \\
$11 \mathrm{~A}$ & .1400 & .7610 \\
$12 \mathrm{E}$ & .4182 & .7410 \\
$13 \mathrm{~A}$ & .2193 & .7558 \\
$14 \mathrm{E}$ & .3042 & .7496 \\
$15 \mathrm{~N}$ & .3833 & .7436 \\
$16 \mathrm{E}$ & .4154 & .7421 \\
$17 \mathrm{~A}$ & .3895 & .7429 \\
$18 \mathrm{E}$ & .4119 & .7419 \\
$19 \mathrm{~N}$ & .3110 & .7492 \\
$20 \mathrm{E}$ & .4651 & .7366 \\
\hline $\mathrm{A}$ & &
\end{tabular}

Alfa $=.7581$

$\mathrm{A}=$ Argumentativo $\mathrm{E}=$ Expositivo $\mathrm{N}=$ Narrativo 
Tabla 4

Matriz de correlaciones

Inter-ítems / puntaje total de la prueba de comprensión lectora inferencial CLI-U (N = 717)

\begin{tabular}{lcccccccc}
\hline & R & IP & T & G & CM & $\begin{array}{c}\text { Exp. } \\
\text { VI-VD U }\end{array}$ & AA & $\begin{array}{c}\text { PT CLI- } \\
U\end{array}$ \\
\hline Resumen & 1.000 & $.272^{* *}$ & $.206^{* *}$ & $.159^{* *}$ & $.138^{* *}$ & $.111^{* *}$ & $.105^{* *}$ & $.523^{* *}$ \\
Idea principal & & 1.000 & $.154^{* *}$ & $.171^{* *}$ & $.129^{* *}$ & $.193^{* *}$ & $.175^{* *}$ & $.521^{* *}$ \\
Título & & & 1.000 & $.133^{* *}$ & $.128^{* *}$ & $.152^{* *}$ & $.189^{* *}$ & $.460^{* *}$ \\
Figura & & & & 1.000 & $.087^{*}$ & $.145^{* *}$ & $.185^{* *}$ & $.522^{* *}$ \\
Concepto de & & & & & & & & \\
motivación & & & & & 1.000 & $.164^{* *}$ & $.182^{* *}$ & $.471^{* *}$ \\
Experimento Vi-Vd & & & & & & 1.000 & $.333^{* *}$ & $.592^{* *}$ \\
Aplicaciones en aula & & & & & & & 1.000 & $.588^{* *}$ \\
Puntaje total CL & & & & & & & & 1.000 \\
\hline
\end{tabular}

** p.<.01

${ }^{*}$ p. $<.05$

Para la determinación de la validez de la prueba se ha adoptado un criterio unitario referido a la validación de constructo con la finalidad de evaluar la exactitud con que la prueba CLI-U mide la comprensión lectora inferencial según la propuesta teórica de Kintsch y Van Dijk. En primer término se analizó si los ítems constituían un dominio representativo del contenido y la fuente empleada fue la correspondencia de opiniones de cinco jueces, quienes coincidieron en un $97 \%$. Asimismo, la correlación inter-ítems y puntaje total en comprensión lectora fueron significativas a un nivel de .01 de confianza, lo que revela la homogeneidad y consistencia interna de la prueba, tal como se observa en la tabla 4.

Con la finalidad de determinar la validez teórica de la prueba CLI-U se utilizó el análisis factorial para apreciar la estructura factorial subyacente de la prueba. En primer término se procedió a examinar la viabilidad del análisis factorial, empleándose tres pruebas, cuyos resultados se aprecian en las tablas $5 \mathrm{y}$ 6 : el determinante de la matriz de correlaciones cuyo valor fue de .640 , mayor a .00001 , lo que permite rechazar que existen variables alta y perfectamente correlacionadas; la medida de adecuación de la muestra de Kaiser-Mayer y Olkin (KMO), cuyo coeficiente fue de .717, aceptable según los valores de Kaiser y el test de esfericidad de Barlett, cuyo valor es 318.671 , significativo al uno por ciento de confianza, este hallazgo permitió rechazar que la matriz de correlaciones era una matriz de identidad, es decir los valores externos a la diagonal no eran iguales a .00. Estos resultados iniciales indican que resulta pertinente la utilización del análisis factorial y que puede conducirnos a resultados satisfactorios. 
Tabla 5

Matriz de correlaciones de la prueba de comprensión lectora inferencial CLI-U (N = 717)

\begin{tabular}{|c|c|c|c|c|c|c|c|c|}
\hline $\mathrm{R}$ & IP & $\mathrm{T}$ & \multicolumn{2}{|c|}{ G } & C M & Ex & $V i-V d A A$ & \\
\hline Resumen (R) & & 1.000 & $.261^{*}$ & .198 * & $.181 *$ & .132 * & $.107^{*}$ & $.107^{*}$ \\
\hline Idea principal (IP) & & & 1.000 & $.150 *$ & .159 * & .134 * & $.171 *$ & $.167^{*}$ \\
\hline Título $(\mathrm{T})$ & & & & 1.000 & .130 * & $.111 *$ & .152 * & $.185^{*}$ \\
\hline Figura (G) & & & & & 1.000 & $.101 *$ & .142 * & .189 * \\
\hline Concepto de motivac & $\mathrm{CM})$ & & & & & 1.000 & .146 * & .169 * \\
\hline Experimento $\mathrm{Vi}-\mathrm{Vd}($ & Vd) & & & & & & 1.000 & .312 * \\
\hline Aplicaciones en aula & & & & & & & & 1.000 \\
\hline
\end{tabular}

${ }^{*} \mathrm{p} .<.01$

Determinante $=.640$

Tabla 6

Medida de adecuación de la muestra de estudiantes universitarios $(\mathrm{N}=717$ )

\begin{tabular}{lcc}
\hline Kaiser, Meyer y Olkin (KMO) & & .717 \\
\hline Test de esfericidad de Bartlett's & Aprox. Chi-Square & 318.671 \\
& g.I. & 21 \\
& Sig. & .000 \\
\hline
\end{tabular}

Tabla 7

Pesos factoriales no rotados y rotados obtenidos en el análisis de los componentes principales de los ítems del CLI-U en la muestra total $(\mathbf{N}=717)$

\begin{tabular}{|c|c|c|c|c|c|c|c|c|c|}
\hline \multirow[b]{2}{*}{ Componente } & \multicolumn{3}{|c|}{ Eigenvalues iniciales } & \multicolumn{3}{|c|}{$\begin{array}{c}\text { Extracción de suma de } \\
\text { cuadrados } \\
\text { Cargas factoriales }\end{array}$} & \multicolumn{3}{|c|}{$\begin{array}{c}\text { Rotación de suma } \\
\text { de cuadrados } \\
\text { Cargas }\end{array}$} \\
\hline & Total & $\begin{array}{c}\% \text { de } \\
\text { varianza }\end{array}$ & $\begin{array}{c}\% \\
\text { acumulado }\end{array}$ & Total & $\begin{array}{c}\% \text { de } \\
\text { varianza }\end{array}$ & $\begin{array}{c}\% \\
\text { acumulado }\end{array}$ & Total & $\begin{array}{c}\% \text { de } \\
\text { varianza }\end{array}$ & $\begin{array}{c}\% \\
\text { acumulado }\end{array}$ \\
\hline 1 & 1.981 & 28.300 & 28.300 & 1.981 & 28.300 & 28.300 & 1.530 & 21.851 & 21.851 \\
\hline 2 & 1.025 & 14.637 & 42.938 & 1.025 & 14.637 & 42.938 & 1.476 & 21.087 & 42.938 \\
\hline 3 & .900 & 12.853 & 55.790 & & & & & & \\
\hline 4 & .871 & 12.437 & 68.227 & & & & & & \\
\hline 5 & .838 & 11.968 & 80.195 & & & & & & \\
\hline 6 & .711 & 10.163 & 90.357 & & & & & & \\
\hline 7 & .675 & 9.643 & 100.000 & & & & & & \\
\hline
\end{tabular}

Método de extracción: Análisis de componentes principales. 
Se han utilizado dos métodos de extracción: el análisis de los componentes principales con estimación de comunalidades y una rotación ortogonal con el método varimax. Por medio del primer método se estimaron los factores de la prueba de comprensión lectora inferencial (CLI-U) en la muestra total de estudiantes universitarios de los dos primeros ciclos. Se observa en la tabla 7 dos factores cuyos eigenvalues para la solución rotada fue de 1.530 para el primer componente y de 1.476 para el segundo, los porcentajes de varianza explicada por cada factor son similares y para los dos factores es el 42,938 por ciento de la variabilidad total, lo que puede interpretarse como un porcentaje moderado para sostener que la prueba mide el constructo de comprensión lectora para la que fue elaborada.

En la tabla 8 se observan los pesos factoriales rotados de los ítems de la prueba CLI-U, mediante el procedimiento ortogonal varimax, que son validados para cada factor, empleándose un índice discriminativo mayor a .40 . La solución factorial dio lugar a dos factores:

El primer factor incluye los ítems resumen, cuyo peso factorial sobre el primer componente es .796; la idea principal, con .647; el título .465 y el gráfico con .442 , a este primer factor (F1) se le ha denominado procesamiento macroestructural o texto base.

El segundo factor comprende los elementos aplicaciones en el aula, con un peso factorial de .768 , identificar la variable independiente y dependiente en un experimento con .754 y enunciar el concepto de motivación, con .409 , a este factor se lo ha denominado modelo de la situación (F2).

Tabla 8

Pesos factoriales rotados de los ítems del CLI-U mediante el método varimax $(\mathrm{N}=717)$

\begin{tabular}{lcc}
\hline & Componentes \\
\hline & 1 & 2 \\
Resumen & .796 & \\
Idea principal & .647 & \\
Título & .465 & \\
Figura & .442 & \\
Aplicaciones en aula & & .768 \\
Experimento Vi-Vd & & .754 \\
Concepto de motivación & & .409 \\
\hline
\end{tabular}

\section{EVALUACIÓN DE LA COMPRENSIÓN LECTORA DEL TEXTO SELECCIONADO DE PSICOLOGÍA}

Con la finalidad de evaluar a los alumnos en la comprensión del texto seleccionado, se analizará en primer lugar los resultados para cada una de las actividades propuestas.

\section{Resumen}

A partir del texto, los alumnos tenían que escoger aquellas ideas o proposiciones que consideren de relevancia y conectarlas entre ellas a fin de que su resumen sea coherente. Para evaluar este ítem se procedió a identificar en cada uno de los resúmenes elaborados 
por los alumnos la presencia de las ideas expuestas en el texto (proposiciones y macroestructura), su coherencia general en la ilación y si no existe distorsión de la información.

El análisis de la coherencia del resumen está basado en la idea principal, la mención del concepto de motivación intrínseca, los experimentos propuestos en el texto y las conclusiones. Se adoptó el criterio de calificar como bueno si había logrado satisfacer todos los requisitos expuestos, regular si no logra todo lo exigido, y malo si no satisface ninguna de las condiciones.

En la figura 2 se aprecia que el $48 \%$ de estudiantes desarrollan un mal resumen y solo el 18 por ciento lo hacen bien y el $25 \%$ regular, es decir no logran explicitar la macroestructura.

Figura 2

Porcentajes de respuestas al resumen $(N=717)$

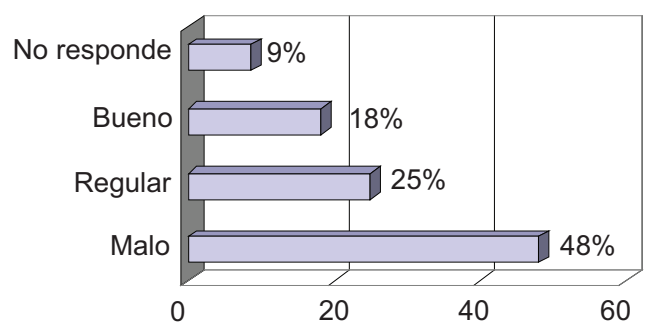

\section{La idea principal}

Las estrategias de identificación de la idea principal y el resumen en realidad son dos actividades cognitivas que pertenecen a una misma especie: el procesamiento macroestructural del texto. Los alumnos debían jerarquizar las ideas con que trabajaron en el resumen y extraer aquellas que consideraron más relevantes, es decir, construir la macroestructura del texto o al menos acercarse a ella. Para evaluar este ítem se ha analizado si los alumnos detectaron correctamente la jerarquía en el texto a través de la idea principal que escribieron. En el texto seleccionado, la idea principal de este se destacó con letra cursiva, pero no en el original, esto ayuda al lector a extraer la idea sin mucha dificultad y constituye un recurso técnico muy efectivo. La macroes-

Figura 3

Porcentajes de respuestas a la idea principal $(\mathrm{N}=717)$

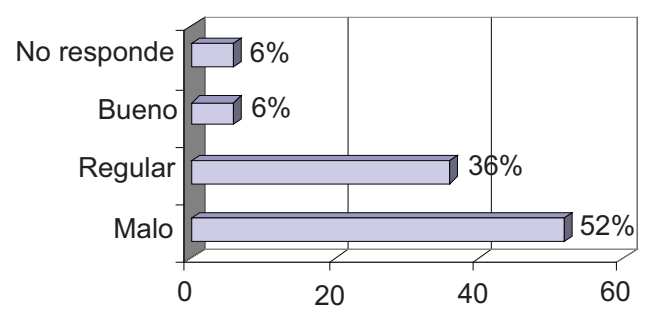


tructura del texto se condensa en la idea: “... los niños encuentran que las tareas realizadas en un contexto rico en fantasía son intrínsecamente atractivas, de modo que proporcionar ese contexto aumentará su motivación intrínseca y mejorará su desempeño" (véase anexo), que se solicita a los estudiantes que reconozcan. Baker (1994) expresa que la capacidad de identificar la idea principal es un aspecto crucial de la comprensión y puede ser considerada una estrategia cognitiva observable. Según la corrección con que expresaron la idea principal, esta fue calificada con la escala tricotómica.

La figura 3 presenta el porcentaje de repuestas a la idea principal, cuya identificación la hizo mal el 52\%, regular el $36 \%$ y bueno solo el $6 \%$.

\section{Título}

El análisis de las pruebas en este caso se aboca a determinar la coherencia entre el título colocado y lo expresado globalmente por el texto. Se ha evaluado como bueno un título que reúne las condiciones señaladas; regular cuando no satisface estrictamente las condiciones, y malo cuando es incoherente. La figura 4 destaca que el $44 \%$ no logró identificar el título y el 50\% lo hizo regular.
Figura 4

Porcentajes de respuestas al título

$(\mathrm{N}=717)$

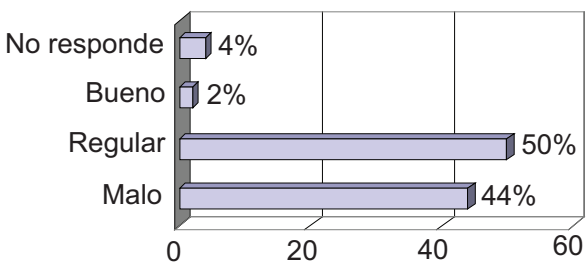

\section{Interpretación gráfica}

La interpretación gráfica debe contener no solo la indicación de la motivación intrínseca sino también de los experimentos reportados para ser evaluada como buena. En los esquemas conceptuales se tiene en cuenta la coherencia interna. En ambos casos se procedió a calificar según los tres niveles expuestos anteriormente.

La figura 5 revela que el $34 \%$ no logró una elaboración visual del texto, bien lo hizo el $25 \%$ y regular el $15 \%$.

\section{Respuestas a un cuestionario sobre el texto}

Para evaluar la comprensión de los estudiantes sobre el texto y comparar luego con los resultados anteriores se formularon tres preguntas referidas al concepto de motivación intrínseca, co- 
Figura 5

Porcentajes de respuestas al gráfico $(\mathrm{N}=717)$

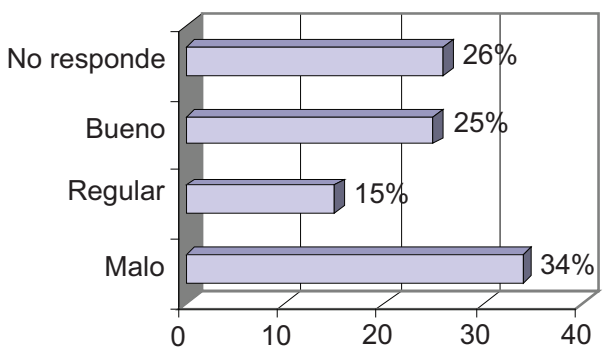

Figura 7

Porcentaje de respuestas al experimento variable independiente - variable dependiente $(\mathrm{N}=717)$
Figura 6

Porcentaje de respuestas al concepto de motivación ( $\mathrm{N}=717$ )

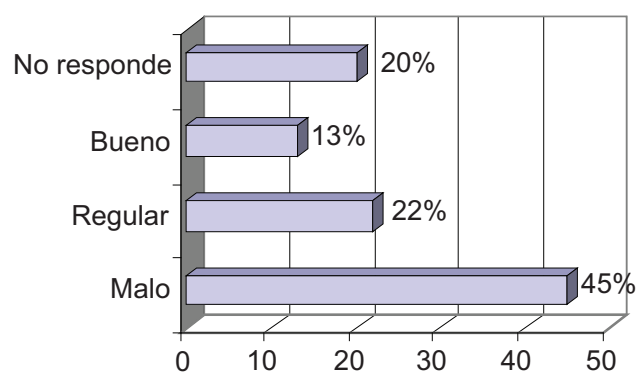

Figura 8

Porcentaje de respuestas a las aplicaciones en el aula $(\mathbf{N}=717)$
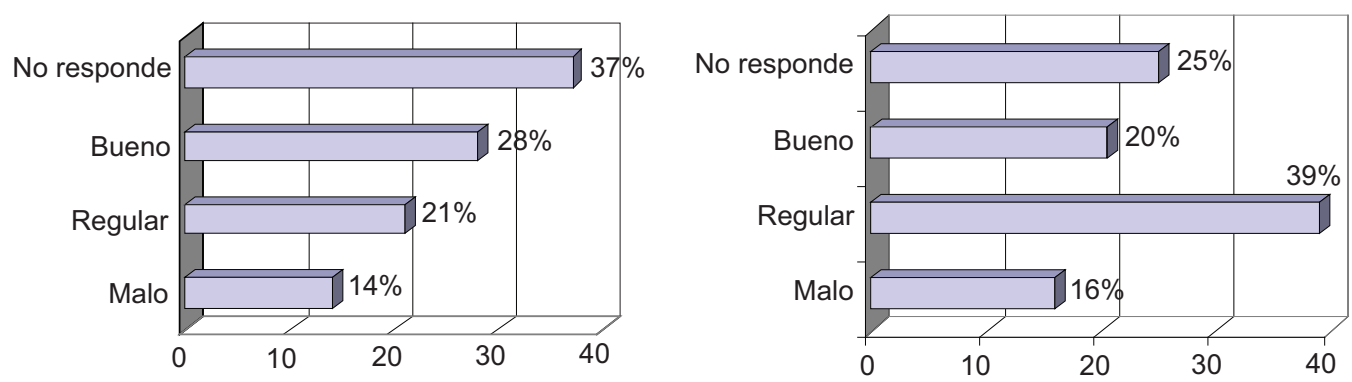

Figura 9

Porcentaje de respuestas a las estrategias que te han ayudado más para responder $(\mathrm{N}=717)$

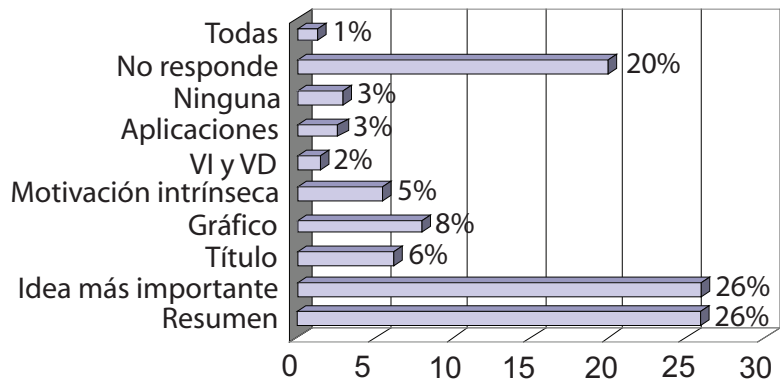


nocimiento de la identificación de las variables involucradas en uno de los experimentos y la relevancia de estos en cuanto a sus aplicaciones en el área educativa. La calificación se realizó siguiendo los criterios formulados anteriormente.

El 45\% no logró conceptualizar la motivación (figura 6); el 14\% no logró identificar la variable independiente y dependiente de un experimento y el $37 \%$ no respondió, lo hizo bien el $28 \%$ (figura 7) y respecto a las aplicaciones (figura 8) que se podían inferir de los experimentos en el aula el 39\% lo hizo regular y bien el $20 \%$.

\section{Opiniones}

Se solicitó a los alumnos que identificaran aquellas actividades que le ayudaron a comprender el texto. Los resul- tados obtenidos se presentan en la figura 9: el 26\% contestó que la idea más importante así como también el resumen les ayudó a comprender más el texto.

\section{ANÁLISIS COMPARATIVO DE LOS RENDIMIENTOS EN COMPRENSIÓN LECTORA INFERENCIAL}

Cuando se compara el rendimiento de los estudiantes en comprensión lectora inferencial (tabla 9) considerando el sexo, las mujeres presentan media de rangos más altas que los varones, en el factor 1: texto base y en el modelo de la situación. La edad no establece diferencias en los aspectos evaluados pero sí la carrera, los estudiantes de Humanidades rinden más que los de Ingeniería y Negocios, no existiendo diferencias entre estos dos últimos.

Tabla 9

Media de rangos para el procesamiento macroestructural (F1), modelo de la situación (F2) y comprensión lectora inferencial (CLI-U)

\begin{tabular}{|c|c|c|c|c|c|c|}
\hline \multicolumn{3}{|l|}{ Variables } & \multirow{2}{*}{$\begin{array}{l}\text { Media de } \\
\text { rangos para el } \\
\text { factor } 1\end{array}$} & \multirow{2}{*}{$\begin{array}{l}\text { Media de } \\
\text { rangos para el } \\
\text { factor } 2\end{array}$} & \multirow{2}{*}{$\begin{array}{l}\text { Media de } \\
\text { rangos para el } \\
\text { PT CLI-U }\end{array}$} & \multirow[b]{2}{*}{ Sig. } \\
\hline & & $\mathrm{N}$ & & & & \\
\hline \multirow[t]{2}{*}{ Sexo } & Masculino & 388 & 327.63 & 337.86 & 326.80 & .000 \\
\hline & Femenino & 329 & 395.99 & 383.93 & 396.98 & .002 \\
\hline \multirow[t]{2}{*}{ Edad } & 16-17 años & 353 & 346.52 & 351.44 & 347.67 & .107 \\
\hline & 18 y más & 364 & 371.10 & 366.34 & 369.98 & .326 \\
\hline \multirow[t]{3}{*}{ Carrera } & Humanidades & 347 & 423.12 & 401.25 & 426.19 & .000 \\
\hline & Negocios & 154 & 296.51 & 324.68 & 297.13 & .000 \\
\hline & Ingeniería & 216 & 300.55 & 315.59 & 295.17 & .000 \\
\hline
\end{tabular}


En la tabla 10 se puede observar la comparación entre las submuestras de estudiantes que llevaban el curso por primera vez, los que pasaron y los repitentes, siendo las diferencias significativas con relación a los que habían aprobado la asignatura, estos obtuvieron medias de rangos más elevadas en las siguientes actividades: realizar un resumen, identificar la idea principal, dar un título a lo leído, interpretar gráficamente el texto, responder a preguntas específicas sobre el contenido y no se dieron diferencias entre los que pasaron el curso y los repitentes.

Tabla 10

Media de rangos para la comprensión lectora inferencial (CLI-U) y las veces que el alumno llevó la asignatura de psicología

\begin{tabular}{|c|c|c|c|}
\hline & Veces que cursó Psicología & $N$ & Media de rangos \\
\hline Resumen & $\begin{array}{c}\text { Primera vez } \\
\text { Pasaron el curso } \\
\text { Repitentes }\end{array}$ & $\begin{array}{c}387 \\
254 \\
76\end{array}$ & $\begin{array}{l}329.71 \\
421.58 \\
299.01\end{array}$ \\
\hline Idea principal & $\begin{array}{c}\text { Primera vez } \\
\text { Pasaron el curso } \\
\text { Repitentes }\end{array}$ & $\begin{array}{c}387 \\
254 \\
76\end{array}$ & $\begin{array}{l}340.30 \\
396.71 \\
328.18\end{array}$ \\
\hline Título & $\begin{array}{c}\text { Primera vez } \\
\text { Pasaron el curso } \\
\text { Repitentes }\end{array}$ & $\begin{array}{c}387 \\
254 \\
76\end{array}$ & $\begin{array}{l}340.36 \\
394.69 \\
334.63\end{array}$ \\
\hline Figura & $\begin{array}{c}\text { Primera vez } \\
\text { Pasaron el curso } \\
\text { Repitentes }\end{array}$ & $\begin{array}{c}387 \\
254 \\
76\end{array}$ & $\begin{array}{l}343.99 \\
389.50 \\
333.47\end{array}$ \\
\hline Concepto de motivación & $\begin{array}{c}\text { Primera vez } \\
\text { Pasaron el curso } \\
\text { Repitentes }\end{array}$ & $\begin{array}{c}387 \\
254 \\
76\end{array}$ & $\begin{array}{l}337.28 \\
407.34 \\
308.03\end{array}$ \\
\hline Experimento $\mathrm{Vi}-\mathrm{Vd}$ & $\begin{array}{c}\text { Primera vez } \\
\text { Pasaron el curso } \\
\text { Repitentes }\end{array}$ & $\begin{array}{c}387 \\
254 \\
76\end{array}$ & $\begin{array}{l}343.70 \\
399.38 \\
301.93\end{array}$ \\
\hline Aplicaciones en aula & $\begin{array}{c}\text { Primera vez } \\
\text { Pasaron el curso } \\
\text { Repitentes }\end{array}$ & $\begin{array}{c}387 \\
254 \\
76\end{array}$ & $\begin{array}{l}352.42 \\
384.61 \\
306.91\end{array}$ \\
\hline PT Factor 1 & $\begin{array}{c}\text { Primera vez } \\
\text { Pasaron el curso } \\
\text { Repitentes }\end{array}$ & $\begin{array}{c}387 \\
254 \\
76\end{array}$ & $\begin{array}{l}321.01 \\
437.67 \\
289.54\end{array}$ \\
\hline PT Factor 2 & $\begin{array}{c}\text { Primera vez } \\
\text { Pasaron el curso } \\
\text { Repitentes }\end{array}$ & $\begin{array}{c}387 \\
254 \\
76\end{array}$ & $\begin{array}{l}337.41 \\
416.74 \\
275.99\end{array}$ \\
\hline Puntaje total CL & $\begin{array}{c}\text { Primera vez } \\
\text { Pasaron el curso } \\
\text { Repitentes }\end{array}$ & $\begin{array}{c}387 \\
254 \\
76\end{array}$ & $\begin{array}{l}320.87 \\
445.53 \\
263.95\end{array}$ \\
\hline
\end{tabular}


Con relación al procesamiento macroestructural (Factor 1) y el modelo de la situación (Factor 2), los que pasaron el curso obtuvieron medias de rangos más elevadas en ambos factores que los que llevaban el curso por primera vez y que los repitentes. Asimismo, los que llevaron la asignatura de Psicología por primera vez obtuvieron logros más elevados que los repitentes, excepto en comprensión lectora total.
Cuando se comparan a los alumnos de rendimiento alto y bajo en la asignatura de Psicología en la tabla 11 se observa que los primeros realizan de manera más eficiente las actividades que tienen que ver con la identificación de las variables independientes y dependientes en un experimento, las inferencias con respecto a las aplicaciones en el aula; el macroprocesamiento estructural (F1) y el modelo de la situación F2, lo mismo que en comprensión lectora total.

Tabla 11

Media de rangos en CLI-U para los grupos de rendimiento alto $(\mathrm{N}=105)$ y bajo $(N=161)$ en el curso de Psicología

\begin{tabular}{lccc}
\hline & Rendimiento en Psicología & Media de rangos & Sig. \\
\hline Resumen & Bajo & 127.19 & .076 \\
Alto & 143.18 & \\
Idea principal & Bajo & 133.07 & .899 \\
\multirow{2}{*}{ Título } & Alto & 134.17 & \\
& Bajo & 128.93 & .163 \\
Figura & Alto & 140.50 & \\
& Bajo & 128.87 & .174 \\
Concepto de motivación & Alto & 140.60 & \\
& Bajo & 130.03 & .315 \\
Experimento Vi-Vd & Alto & 138.81 & \\
& Bajo & 125.51 & .024 \\
Aplicaciones en aula & Alto & 145.76 & \\
& Bajo & 125.38 & .022 \\
PT Factor 1 & Alto & 145.96 & \\
& Bajo & 125.65 & .037 \\
PT Factor 2 & Alto & 145.54 & \\
& Bajo & 123.74 & .009 \\
PT CLI-U & Alto & 148.46 & \\
& Bajo & 121.94 & .002 \\
\hline
\end{tabular}




\section{RELACIÓN ENTRE LA COMPRENSIÓN} LECTORA INFERENCIAL Y OTRAS VARIABLES

Al examinar las diversas actividades vinculadas con la comprensión lectora inferencial y las estrategias que más ayudaron a los estudiantes a comprender el texto, se ha podido constatar una correlación significativa al uno por ciento pero inversa, tal como se presenta en la tabla 12. Lo que revelaría que la actividad que en opinión del estudiante más le había apoyado en la comprensión del texto, se encontraba asociada a un menor rendimiento en comprensión lectora.

\section{Tabla 12}

Relación entre la comprensión lectora inferencial y la estrategia elegida que ayudó a comprender el texto

\begin{tabular}{lc}
\hline Variables & $\begin{array}{c}\text { ¿Cuál de las estrategias } \\
\text { te ha ayudado más? }\end{array}$ \\
\hline Resumen & $-.120^{*}$ \\
Idea principal & $-.158^{*}$ \\
Título & $-.141^{*}$ \\
Figura & $-.134^{*}$ \\
Concepto de motivación & $-.200^{*}$ \\
Experimento Vi-Vd & $-.239^{*}$ \\
Aplicaciones en aula & $-.244^{*}$ \\
Puntaje total CLI-U & $-.318^{*}$ \\
PT Factor 1 & $-.207^{*}$ \\
PT Factor 2 & $-.322^{*}$
\end{tabular}

* p. $<.01$
En la tabla 13 se aprecia una relación significativa entre la autopercepción de su rendimiento en lengua y literatura y la solución de las actividades exigidas en la comprensión del texto en psicología. No ocurre lo mismo con la autopercepción del rendimiento en matemáticas y en el caso de la autopercepción del rendimiento en ciencias solo existen asociaciones significativas con la identificación de la idea principal y el título (procesamiento macroestructural del texto) y así mismo con el señalamiento de la VI y VD de un experimento (modelo de la situación).

Tabla 13

Correlaciones entre la autopercepción de rendimiento en secundaria y la comprensión lectora inferencial (CLI-U)

\begin{tabular}{lccc}
\hline \multicolumn{4}{c}{ Rendimiento en } \\
\hline & $\begin{array}{c}\text { Lengua y } \\
\text { Literatura }\end{array}$ & Matemáticas & Ciencias \\
\hline Resumen & $.234^{* *}$ & .011 & .063 \\
Idea principal & $.167^{* *}$ & .015 & $.084^{* *}$ \\
Título & $.172^{* *}$ & -.005 & $.086^{* *}$ \\
Figura & $.090^{*}$ & -.025 & .038 \\
Concepto de & & & \\
$\quad$ motivación & $.112^{* *}$ & .016 & -.005 \\
Experimento Vi-Vd & $.163^{* *}$ & .060 & $.103^{* *}$ \\
Aplicaciones en aula & $.214^{* *}$ & -.019 & .031 \\
Puntaje total CL & $.309^{* *}$ & -.001 & $.103^{* *}$ \\
PT Factor 1 & $.272^{* *}$ & -.008 & $.104^{* *}$ \\
PT Factor 2 & $.231^{* *}$ & .024 & .070 \\
& & & \\
\hline$* *$ p. $<.01$ & & & \\
${ }^{*}$ p. $<.05$ & & & \\
\end{tabular}


Tabla 14

Correlación entre el rendimiento de la asignatura de Psicología y la comprensión lectora evaluada a través de la CLI-U y de GUB

\begin{tabular}{lccc}
\hline & Puntaje total CLI-U & PT GUB & Psicología \\
\hline Puntaje total CLI - U & 1.000 & $.238^{* *}$ & $.117^{*}$ \\
PT GUB & & 1.000 & $.139^{* *}$ \\
Psicología & & & 1.000 \\
\hline${ }^{* *}$ p. $<.01$ & & \\
$*$ p. $<.05$ & &
\end{tabular}

Finalmente, en la tabla 14 se observa una relación significativa entre las pruebas de comprensión lectora CLI-U y GUB, lo que revela su validez. Así mismo, se encuentra correlación significativa entre la comprensión lectora y el rendimiento en psicología.

\section{Discusión}

El análisis psicométrico arroja resultados consistentes y válidos en relación con la prueba de comprensión lectora inferencial (CLI-U), siendo una técnica muy eficaz para la recolección de los datos en el presente estudio. El análisis factorial de la prueba, mediante el procedimiento de rotación ortogonal varimax, dio lugar a una solución factorial de dos factores. El primer factor, F1, que se ha denominado procesamiento macroestructural, y el segundo factor, F2, modelo de la situación.

F1 tiene que ver con la representación semántica de naturaleza abstracta y global del texto, supone una reducción selectiva de la información, reteniendo el sentido del texto, formando una microestructura que permite comprender el texto. F2 tiene que ver con el modelo de la situación que es la representación cognitiva de los acontecimientos, acciones, personas y, en general, de la situación sobre la que trata el texto (Maturano et al., 2002). Representar la situación que describe el texto supone incorporar gran cantidad de elementos no mencionados explícitamente en el texto, provenientes de la base de conocimientos del lector. La información procedente del texto se integra con dicho conocimiento, dando como resultado una representación más rica que la base del texto.

Los resultados demuestran que la mayoría de los alumnos no lograron desarrollar un buen resumen; como se sabe, el resumen constituye una poderosa estrategia, porque quien lo elabora se obliga a profundizar y reflexionar de manera consciente sobre la macroestructura y la superestructura del texto, sobre el modelo de la situación y a reformular con los códigos y el vocabulario personal aquello que el autor nos quiso decir por medio del texto. 
Tampoco identificaron la idea principal a pesar de los marcadores intratextuales (que aparece en letra cursiva) lo que les hubiese permitido mejorar su comprensión. La identificación de la idea principal involucra las siguientes actividades: a) construir una representación global del texto que implica el tema, es decir, a partir del texto, los alumnos debían escoger aquellas ideas o proposiciones que consideraron de relevancia y conectarlas entre ellas a fin de que su síntesis fuese coherente; b) hacer juicios sobre la importancia de la información y reducir lo trivial, secundario y redundante; y c) consolidar la idea principal, después de hacer un análisis reflexivo sobre el peso de aquellas ideas que se consideraron relevantes.

Las investigaciones realizadas señalan que los lectores expertos aprenden a identificar las ideas principales de un texto (Winograd \& Bridge, 1990) debido a su experiencia previa con multiplicidad de textos, que les ha permitido incrementar su capacidad para identificar las palabras clave o marcadores textuales que se encuentren en ellos, lo cual los conduce a identificar la superestructura textual y, con ello, la idea principal. Además, por el amplio conocimiento que poseen sobre el tema y que activan durante la lectura, les lleva a diferenciar la información principal de la secundaria.

Las estrategias de identificación de la idea principal, el resumen y el colo- car el título al texto en realidad son actividades cognitivas que pertenecen a un mismo género: el procesamiento macroestructural del texto. Lo mismo puede decirse de la representación visual del texto mediante la utilización de un organizador gráfico, consistente en esquemas conceptuales con coherencia interna. Lo que los alumnos hacen es jerarquizar las ideas con que trabajaron en el resumen y extraer aquella que consideren más relevante, es decir, construir la macroestructura del texto o al menos acercarse a ella, todas estas actividades revelan el grado de comprensión logrado con respecto al contenido leído.

Como se ha podido constatar, los alumnos tuvieron problemas con la construcción de la macroestructura. No llegaron a ser capaces de construir con certeza una representación de la información contenida en él, que le dé sentido. El problema, aquí, consiste en una inadecuada aplicación de los macroprocesos, ya sea por falta de madurez para poder hacerlo o debido al desconocimiento de cómo ejecutarlas en forma correcta.

Otra dificultad estuvo relacionada con la identificación de la estructura formal del texto. No fueron capaces de identificar que las distintas partes del texto conforman una superestructura, que les permitiría, a su vez, perfeccionar su representación macroestructural (resumen). Metafóricamente hablando, puede decirse que algunos alumnos lo- 
graron identificar los enunciados ("árboles") y hasta los enunciados principales del texto; pero no consiguieron percatarse de la estructura global (la superestructura) del que son parte ("bosque").

El uso de un cuestionario formulado con base en el texto fue una eficaz herramienta de aprendizaje y de evaluación de la comprensión y del contenido. Se formularon tres preguntas abiertas, cuyas respuestas tenían que inferirse de la información implícita en el texto, estas tenían un carácter aplicativo, de opinión reflexiva y crítica. Las preguntas estaban referidas al concepto de motivación intrínseca, a la identificación de las variables involucradas en uno de los experimentos y la relevancia de estos en cuanto a sus aplicaciones en el área educativa. No consiguieron así construir el modelo de la situación donde los conocimientos anteriores sobre los eventos descritos en él desempeñan un rol decisivo.

Otro problema estuvo referido al proceso de comprensión directamente relacionada con la aplicación de las estrategias autorreguladoras. Por ejemplo, no supieron autosupervisar en forma eficaz las estrategias específicas de lectura que se ejecutan y, tampoco evaluar el proceso global o el producto alcanzado al término de la actividad de comprensión, es decir, no lograron diferenciar cuándo se está comprendiendo y cuándo no (metaignorancia). Se halló una correlación significativa pero negativa al relacionar la estrategia preferida por el estudiante para comprender el texto y la comprensión de este. En otras palabras, utilizan estrategias que no le ayudan a la comprensión.

El sexo establece claras diferencias a favor de las mujeres y cuando se hacen las comparaciones considerando las carreras son los de Humanidades los que alcanzan mejores logros en comprensión lectora inferencial global así como también en el macroprocesamiento estructural (F1) y en el modelo de la situación (F2), comparados con los de Negocios e Ingeniería, este resultado es explicable por la naturaleza de las aptitudes desarrolladas por los estudiantes en función del campo elegido y de sus intereses.

En cuanto al rendimiento de la asignatura de Psicología, los que pasaron el curso exhiben más altos logros en la comprensión lectora inferencial, tanto en el macroprocesamiento estructural como en el modelo de la situación, lo que también se refleja en la relación significativa entre el rendimiento en el curso de Psicología y la comprensión lectora. Esto quizás se atribuya al contenido de esta materia, muy vinculado al texto cuyas actividades tenían que desarrollar. Los que fracasaron en la asignatura no pudieron elaborar el modelo de la situación donde los conocimientos previos y las estructuras conceptuales anteriores sobre los eventos descritos en él juegan un papel crucial. 
En resumen, estas explicaciones se reflejan en los resultados alcanzados en la prueba de comprensión lectora inferencial, que mediante el procedimiento del análisis factorial da lugar a una solución de dos factores: el F1, procesamiento macroestructural que reúne las siguientes actividades: realizar un resumen, identificar la idea principal, colocar un título al texto leído y hacer uso de organizadores gráficos; y el F2, modelo de la situación, donde intervienen de manera decisiva los conocimientos previos y las actividades inferenciales que el lector realiza y que no aparecen expresamente señaladas por el autor en el texto. Como se ha podido apreciar, los estudiantes de los dos primeros ciclos de estudios generales de una universidad particular fracasan en los aspectos mencionados y esto se encuentra asociado a sus bajos logros en la asignatura de Psicología.

Las estrategias de aprendizaje utilizadas por los estudiantes han sido limitadas en parte por las actividades que la prueba de comprensión lectora demandaba. Es por esta razón que las conclusiones que se extraen se refieren a ellas, aun cuando los alumnos puedan haber hecho uso de otras estrategias que no fueron exploradas en este estudio.

Resulta evidente que, en general, hay una pobre comprensión del texto de parte de los estudiantes evaluados, sin embargo, esta actividad debería ser guiada y controlada por el propio lector, quien debe construir sus conoci- mientos en base a la información previa y lo que le proporciona el texto. No obstante, esta construcción no surge por iniciativa propia del estudiante, por ello se requiere la intervención de los docentes, quienes deben promover su acercamiento al texto y enseñarles estrategias de comprensión lectora para el aprendizaje de los textos expositivos de las asignaturas que tienen a su cargo.

Finalmente, se debería encontrar la forma de trabajar la comprensión de textos en los estudiantes de matemáticas y ciencias, quienes obtuvieron los menores puntajes, dado que estos también requieren comprender problemas matemáticos que son expresados en enunciados verbales.

\section{ReFERENCIAS}

Baker, L. (1994). Metacognición, lectura y educación científica. En: C. Minnick Danta \& D. E. Alvermann (Comp.). Una didáctica de las ciencias, procesos y aplicaciones. Buenos Aires: Aique.

Balluerka, N. (1995). Cómo mejorar el estudio y aprendizaje de textos de carácter científico. Bilbao: Servicio Editorial de la Universidad del País Vasco.

Bassols, M. \& Torret, A. (1997). Modelos textuales. Barcelona: Octaedro.

Cope (1978) \& Tinto (1975). [en línea]. $<$ http://www.ponce.inter.edu/cai/tesis/1 vera/cap1.htm>.

De Vega, M.; Díaz, J. M. \& León, I. (1999). Procesamiento del discurso. En: M. De 
Vega \& F. Cuetos. (Coords.). Psicolingüística en español. Madrid: Trotta.

De Vega, M. (1986). Introducción a la psicología. México: Alianza Editorial.

Díaz-Barriga, F. \& Hernández, G. (2001). Estrategias docentes para un aprendizaje significativo. México: McGrawHill.

García, J. A.; Elosúa, M. R.; Gutiérrez, F.; Luque, J. L. \& Gárate, M. (1999). Comprensión lectora y memoria operativa. Barcelona: Paidós.

González, R. (1996). Lectoescritura: Aspectos cognitivos y evolutivos. Cuadernos Cedehum 5. Lima: Sergrafín EIRL.

González, R. (1998). Comprensión lectora en estudiantes universitarios iniciales. Persona, 1, 9-40.

González, R. (2000). Factores que influyen en el desempeño matemático de estudiantes universitarios iniciales. Persona, 3, 11-26.

González, R. (2001). El papel del componente superestructural en la elaboración de las macroestructuras. Persona, 4, 53-72.

González, R. (2002). Adquisición del lenguaje y desarrollo del pensamiento. Influencia de estos procesos en aprendizajes posteriores. En: L. M. Saravia (Ed.) Lectora comprensiva temprana. Para que leamos desde la infancia, mucho más y mejor. Lima: Ministerio de Educación, GTZ Cooperación Técnica Alemana.

González, R. (2005). Comprensión lectora en estudiantes universitarios iniciales. En: Problemas psicolingüísticos en el Perú. Evidencia empírica. En edición.
Gunning, T. (1996). Creating reading instruction for all children. MA: Allyn \& Bacon.

Horowitz, R. (1985). "Patterns of Texts. Part I". Journal of Reading, 28(5), 448454.

Irvin, J. (1998). Reading and the middle schoool student: strategies to enhance literacy, 2nd. Ed. Boston: Allyn \& Bacon.

Kintsch, W. (1998). Comprension: A paradigm for cognition. Cambridge: Cambridge University Press.

Kintsch, W. \& Van Dijk, T. A. (1978). Toward a model of text comprehension and production. Psychological Review, 85, 363-394.

Luria, A. (1979). Conciencia y lenguaje. Lima: Pablo del Río.

Maturano C.; Soliveres, M. A. \& Macías, A. (2002). Estrategias cognitivas y metacognitivas en la comprensión de un texto de Ciencias. Revista Enseñanza de las Ciencias 20(3), 415-425.

MacNamara, T. P., Millar, D. L. y Bransford, J. D. (1991). Mental models and reading comprenhension. En: R. Barr; M. L. Kamil; P. B. Mosenthal. \& P. D. Pearson (Eds.). Handbook of reading research (v. II). Nueva York: Longman.

Mayer, R. E. (1986). Pensamiento, resolución de problemas y cognición. Barcelona: Paidós.

Meyer, J. F. (1984). Texts dimensions and cognitive processing. En: H. Mandl, N. Stein y T. Trabasso (Eds.). Learning and comprehension of texts. Hillsdale, N.J.: Earlbaum. 
Paris, S. G.; Wasik, B. A. \& Turner, J. C. (1991). The development of strategic readers. En: R. Barr; M. L. Kamil; P. B. Mosenthal \& P. D. Pearson (Eds.). Handbook of reading reaserch (vol. II). Nueva York: Longman.

Pressley, M. (1999). Cómo enseñar a leer. Barcelona: Paidós.

Renner (1976). [en línea]. <http://www. ponce.inter.edu/cai/tesis/lvera/cap1. htm>.

Richgels, D. J.; Mcgee, L. M. \& Slaton, E. A. (1990). Cómo enseñar la estructura del texto expositivo en la lectura y la escritura. En: K. D. Ruth (Ed.). El texto expositivo. Buenos Aires: Aique.

Sánchez, E.; Rosales, J. \& Cañedo, J. (1995). La intervención educativa en la comprensión lectora de textos: Más allá de la instrucción en habilidades cognitivas. En: M. Carretero; J. Almaráz \& P. Fernández (Eds.). Razonamiento $y$ comprensión. Madrid: Trotta.

Sánchez, E. (1993). Los textos expositivos. Estrategias para mejorar su comprensión. Madrid: Santillana.

Sánchez, E. (1998). Comprensión y redacción de textos. Barcelona: Edebé.

Sánchez, E. (1998). Comprensión y redacción de textos: Dificultades y ayudas. Barcelona: Edebé.

Slater, W. H. \& Graves, M. F. (1990). Investigaciones sobre el texto expositivo: Aportes para los docentes. En: K. D. Ruth (Comp.). El texto expositivo. Es- trategias para su comprensión. Buenos Aires: Aique.

Ugarriza, N. (2004). Relación entre el componente superestructural y los estilos de aprendizaje de los alumnos universitarios. Universidad Ricardo Palma, CIURP, Lima. (Material inédito)

Van Dijk, T. (1983). La ciencia del texto. Barcelona: Paidós.

Van Dijk, T. (1993). Discourse structures and ideological structures. Paper for the International AILA Congress, Amsterdam.

Van Dijk, T. (1993-1994). Modelos en la memoria. El papel de las representaciones de la situación en el procesamiento del discurso. Revista Latina de Pensamiento y Lenguaje, 2(1), 39-55.

Van Dijk, T. \& Kintsch, W. (1983). Strategies of discourse comprehension. New York: Academic Press.

Vidal-Abarca, E. (2000). Las dificultades de comprensión I: Diferencias en procesos de comprensión entre lectores normales y con dificultades de comprensión. En: A. Miranda; E. VidalAbarca \& M. Soriano (Coords.). Evaluación e intervención Psicoeducativa en dificultades de aprendizaje. Madrid: Pirámide.

Winograd, T. \& Bridge, C. A. (1990). La comprensión de la información importante en prosa. En: J. F. Baumann (Ed.). La comprensión lectora (cómo trabajar la idea principal en el aula). Madrid: Visor. 


\section{ANEXO}

\section{Ficha de datos personales DATOS GENERALES}

Apellidos y nombres:

Edad: Sexo: M ( ) F ( ) Carrera:

Código:

Ciclo:

1. ¿Repetiste en secundaria algún año escolar? sí ( ) NO ( )

2. En secundaria, ¿cómo fue tu calificativo en los siguientes cursos?

$\begin{array}{lllll}\text { Lengua y Literatura } & \text { Muy bueno ( ) } & \text { Bueno ( ) } & \text { Regular ( ) } & \text { Malo ( ) } \\ \text { Matemáticas } & \text { Muy bueno ( ) } & \text { Bueno ( ) } & \text { Regular ( ) } & \text { Malo ( ) } \\ \text { Ciencias } & \text { Muy bueno ( ) } & \text { Bueno ( ) } & \text { Regular ( ) } & \text { Malo ( ) }\end{array}$

3. Si llevas actualmente el curso de Psicología General responde si es por:

Primera vez ( ) Segunda vez ( ) Tercera vez ( )

4. Solo en el caso de que estés en el curso de Sociología o Psicología Social, responde a la siguiente pregunta: ¿Cuántas veces llevaste el curso de Psicología General?

Una vez ( ) Dos veces ( ) Tres veces ( )

\section{PRUEBA DE COMPRENSIÓN DE TEXTOS}

\section{PRIMERA PARTE \\ Instrucciones generales}

Lee atentamente el texto las veces que creas conveniente para responder a las preguntas que se presentan a continuación. Lee las preguntas con detenimiento, trabaja con cuidado y escribe tus respuestas en la hoja adjunta. No olvides numerar tus respuestas a cada pregunta.

A menudo se ha afirmado que el aprendizaje es, o por lo menos debería ser, divertido. Un corolario es que los procedimientos del salón de clases que los niños disfrutan facilitarán su dominio de nuevas ideas y conceptos. En otras palabras, cuando los niños disfrutan las actividades que ocurren en sus grupos quedarán intrínsecamente motivados y mejorará su aprendizaje. ¿Es esto cierto? La investigación realizada hace poco por Leeper y sus colegas (Leeper \& Córdova, 1992; Leeper \& Hoddell, 1992) sugiere que sí. Más aún, esta investigación proporciona información importante sobre procedimientos específicos que los maestros pueden usar para mejorar la motivación intrínseca de los niños.

La base de dichos estudios es la idea de que los niños encuentran que las tareas realizadas en un contexto rico en fantasía son intrínsecamente atractivas, de modo que proporcionar ese contexto aumentará su motivación intrínseca y mejorará su desempeño. Para probar esta predicción general, Leeper y sus colaboradores han realizado una serie de estudios en los que utilizan juegos educacionales computarizados.

Como un ejemplo de esta investigación, considere un estudio conducido por Parker \& Leeper (1992), en que los niños aprendieron a usar un sistema de gráficas por computadora en una con- 
dición motivacional enriquecida por la fantasía o en un contexto que carecía de dicho enriquecimiento. Existían tres versiones de la condición de fantasía. En una, los estudiantes eran animados a imaginar que eran piratas que buscaban un tesoro escondido. En otra, eran detectives a la búsqueda de criminales. En la tercera, eran astronautas que buscaban nuevos planetas. En la condición de control, en contraste, no se proporcionaba ningún contexto de fantasía. En los cuatro grupos, los niños realizaban las mismas actividades: aprender a dibujar líneas que conectaran varios objetos, arreglar un paso a través de una serie de laberintos, construir formas geométricas simples. Los investigadores midieron la motivación para realizar esas tareas pidiendo a los niños que calificaran el atractivo de las mismas. Como se predijo, los estudiantes dieron puntuaciones muchos mayores a los tres juegos de fantasía que a la condición en que esta no existía.

El aprendizaje fue medido por pruebas escritas sobre el dominio que los estudiantes tenían del programa de gráficas, que fueron aplicadas en seguida del estudio o dos semanas más tarde. Una vez más, los resultados mostraron una ventaja impresionante a favor de la versión que usó la fantasía; tanto los chicos como las niñas tuvieron un desempeño mucho mejor después de jugar con esas versiones que después del juego sin fantasía.

En una investigación de seguimiento un tipo diferente de juego, uno que se parecía al juego “¿Quién es el culpable?”, Leeper y sus colaboradores obtuvieron información de las causas por las que los niños aprenden más de los juegos ricos en fantasía. Al parecer, usaron estrategias más eficientes para la solución de problemas y formularon hipótesis de manera más sistemática cuando jugaban en un contexto rico en fantasía en el que eran detectives, que un contexto no enriquecido.

Tal vez lo más importante de todo sea que cuando se ofreció a los niños recompensas extrínsecas (un premio de la caja misteriosa del experimentador) por resolver el acertijo, su desempeño decayó bruscamente. Utilizaron estrategias de solución de problemas más simples, formularon hipótesis de manera menos sistemática y se percibieron como menos competentes para realizar la tarea.

Las implicaciones de esos hallazgos para la educación en el salón de clases parecen ser evidentes (Leeper \& Córdova, 1992): Bajo condiciones apropiadas, aumentar la motivación intrínseca de los estudiantes puede mejorar su aprendizaje de conceptos o materiales importantes. Puesto en otras palabras, en la medida en que los maestros logren cambiar el trabajo en juego, puede también aumentar sustancialmente el aprendizaje y el desempeño de sus alumnos.

\section{Preguntas}

1. Elabora un resumen de lo que has leído.

2. ¿Cuál es la idea más importante que el autor utiliza o sugiere para explicar el tema?

3. ¿Qué título le pondrías al texto que has leído?

4. Expresa mediante un gráfico lo que te sugiere el texto.

5. Responde a las siguientes preguntas:

a) ¿Qué entiendes por motivación intrínseca?

b) En el experimento de Parker y Leeper (1992), ¿cuál es(son) la(s) variable(s) independiente(s) y la(s) variable(s) dependiente(s)?

c) Con base en los experimentos presentados en el texto, ¿qué aplicaciones, según tu criterio, pueden ser útiles para un maestro en el aula?

6. ¿Cuál de las preguntas anteriores te ha ayudado a comprender mejor el texto? 


\section{SEGUNDA PARTE}

Intrucciones

En esta prueba se presentan diferentes neologismos, insertos en textos compuestos por varias (6) frases cada uno. La tarea es inferir el significado de cada neologismo con una palabra existente en nuestro idioma. Escriba la palabra en el lugar correspondiente de la hoja de respuestas. No escriba en este material.

A continuación le damos un ejemplo:

Las TUBRAS deben usarse para curar. Las TUBRAS no deben usarse sin receta. El uso indiscriminado de TUBRAS puede acarrear trastornos. Los médicos recomiendan no abusar de las TUBRAS. La venta de TUBRAS está sometida a restricciones legales. Quien vende las TUBRAS indiscriminadamente corre peligro de ser enjuiciado. La respuesta es MEDICINA. Escriba en la hoja de respuestas la palabra MEDICINA al lado de la palabra EJEMPLO. Trabaje rápido. Tiene aproximadamente 20 minutos para realizar la tarea.

1.

- Nuestro país debe impulsar la DUCRAT en sus instituciones.

- La DUCRAT expresa el punto de vista de la mayoría.

- Para los habitantes de un país es más satisfactorio vivir en DUCRAT.

- La DUCRAT permite la participación libre en los asuntos que nos afectan.

- La DUCRAT previene imposiciones y abusos.

- La DUCRAT es necesaria para el desarrollo del país.

2.

- El INCUS es útil cuando hace calor.

- Si cierras el INCUS pierde su utilidad.

- Los INCUS los usan más en Occidente las mujeres que los varones.

- Los INCUS son de tela o papel.

- El INCUS es también utilizado para el galanteo o flirteo.

- En algunos países el movimiento de los INCUS constituye una expresión artística.

3.

- La BURCA debe estar al alcance de todos en una sociedad.

- La BURCA es un factor causal del desarrollo de las habilidades y destrezas.

- La BURCA transmite los valores y normas de una sociedad a los niños.

- Al formar ciudadanos con mayor conciencia cívica, la BURCA impulsa el desarrollo de un país.

- La BURCA facilita la responsabilidad en las personas.

- La BURCA permite una mayor interacción entre niños, facilitando la formación de relaciones interpersonales sanas.

4.

- Ese DRACO es muy inteligente.

- Pero el DRACO se ponía nervioso para los exámenes. 
- A pesar de eso, era el DRACO con mejores notas.

- Cuando empezó a trabajar las notas del DRACO bajaron.

- EI DRACO tuvo que esforzarse el doble para mantener su promedio.

- Al final el DRACO logró mantener su promedio.

5.

- La NIBRA es necesaria ya que permite conocer el mundo para actuar sobre él.

- La NIBRA ayuda a tomar decisiones adecuadas para cada situación.

- A veces la NIBRA es difícil de aceptar y produce angustia.

- La NIBRA permite resolver los problemas de la vida diaria.

- La NIBRA es un componente fundamental de nuestro conocimiento.

- Descubrir la NIBRA es fundamental para la ciencia.

6.

- Con una EMPLA podemos capturar cosas.

- Las EMPLAS las fabrican los hombres pero también algunos animales.

- Con una EMPLA no puedes retener el agua.

- En un gran número de ocasiones las EMPLAS se utilizan en el agua.

- Cuantos más agujeros tiene una EMPLA mayor es ésta.

- Para no caer en el vacío a veces se usan EMPLAS.

7.

- Para asegurar la armonía en la vida de una sociedad son necesarias las MESTAS.

- Las MESTAS mantienen el orden en la sociedad.

- Las MESTAS se formulan en base a principios de justicia.

- Las MESTAS sancionan las conductas que atentan contra el bien del grupo social.

- Las MESTAS son fundamentales para regular cualquier transacción comercial.

- Las MESTAS pueden revisarse y adaptarse a los cambios de la sociedad.

8.

- Un ALOPE tiene forma cilíndrica.

- Algunos ALOPES pierden su función si se les expone a la luz.

- En un ALOPE caben muchas imágenes.

- Un ALOPE es también la cualidad de ciertos discursos.

- Nadie como una persona quiere ser considerada un ALOPE.

- Las películas tienen varios ALOPES.

9.

- Juan le prestó un FRETO a Manuel.

- Manuel usó el FRETO para hacer un trabajo.

- Era un FRETO muy completo e interesante.

- Manuel terminó su trabajo y le devolvió el FRETO a Juan.

- El trabajo de Manuel obtuvo buena nota gracias al FRETO.

- Juan fue muy amable en prestarle ese FRETO. 
10.

- EI GUNDIS es una propiedad o cualidad.

- EI GUNDIS no puede verse directamente pero sí los movimientos que origina.

- Si un objeto se mueve hacia otro posiblemente el primer objeto tiene GUNDIS.

- EI GUNDIS es una fuerza presente en la naturaleza.

- Una piedra permitió verificar al GUNDIS como fuerza.

- De algunas personas con gran atractivo se dice que tienen GUNDIS.

11.

- Actuando con CUSRA se evitarán conflictos en el trabajo.

- Tener CUSRA es tener seguridad.

- Las personas con CUSRA piensan primero en los demás y luego en ellos mismos.

- Una buena amistad se basa en la CUSRA de ambas partes.

- Una persona con CUSRA tiende siempre a decir la verdad.

- Las parejas deben tratar con CUSRA sus relaciones.

12.

- La AREITIA procede de un animal marino.

- Hay también AREITIAS artificiales.

- Las AREITIAS estando llenas de agujeros pueden, sin embargo, absorber mucho líquido.

- Las AREITIAS son suaves.

- Muchas mañanas utilizamos las AREITIAS.

- Para luchar nadie emplearía AREITIAS.

13.

- EI DICRO mantiene a las personas unidas.

- EI DICRO hace calidad y transparentes las relaciones entre las personas.

- EI DICRO facilita resolver conflictos porque implica tolerancia.

- EI DICRO genera cariño, ternura y afecto hacia los otros.

- El niño necesita mucho DICRO.

- Los esposos deben brindarse DICRO mutuamente.

14.

- EI DRIGO se utiliza para medir.

- EI DRIGO no se ve pero se experimenta a lo largo de la vida.

- Tierra y Sol sirven para establecer los DRIGOS.

- EI DRIGO se divide en partes.

- Cada cuatro DRIGOS una de sus partes aumenta en una unidad.

- En Júpiter los DRIGOS son diferentes en su tamaño.

15.

- En el DARTO había un vendedor de globos.

- Al vendedor se le escaparon todos los globos por el DARTO.

- En el DARTO también había muchos niños.

- Los niños querían atrapar los globos que volaban por el DARTO. 
- Una niña sentada bajo un árbol en el DARTO se tropezó con un globo.

- El globo se reventó asustando a la niña que salió corriendo del DARTO.

16.

- La HULDA sirve para transportar cosas pero a su vez ha de ser transportada.

- Así como una maleta es útil para ciertos viajes, la HULDA también lo es.

- En la montaña vemos en ocasiones gentes con HULDAS.

- La HULDA puede llevarse en la mano, pero no es ese su sitio habitual.

- La HULDA recuerda a los camellos.

- La HULDA nos permite tener las manos libres.

17.

- La GRATE es muy importante para la salud.

- Los padres deben inculcarle la GRATE a sus hijos.

- Sin GRATE podemos contraer enfermedades.

- En la medicina preventiva debe darse prioridad a la GRATE.

- Buenos hábitos de GRATE prolongan la vida.

- Los colegios deben incorporar programas de GRATE en sus asignaturas.

18.

- Las TUPLAS no se comen.

- Las TUPLAS se usan, sin embargo, en los comedores.

- La TUPLA es adecuada para una sola persona.

- Si las TUPLAS fueran seres animados podrían trotar.

- Tiene, esquemáticamente, semejanza con un número y este se usa como expresión tras estar mucho tiempo en una TUPLA.

- La principal función de las TUPLAS es proporcionar descanso y comodidad.

19.

- EI DERME de María se fue de la casa.

- María había vivido seis años con su DERME.

- Cuando su DERME se fue María se puso triste.

- María no supo nada de su DERME por cuatro días.

- Al quinto día su DERME regresó.

- María y su DERME arreglaron sus problemas y siguieron con sus vidas.

20.

- El BIGOJ sirve para sujetar.

- Cuanto más giramos hacia la derecha un BIGOJ más lo introducimos.

- Muchas máquinas tiene BIGOJES.

- La falta de un BIGOJ no se considera una buena cualidad en las personas.

- El BIGOJ tiene una forma cilíndrica.

- No es bueno usar el martillo para introducir BIGOJ. 
Respuestas para el ejemplo:

Hoja de respuestas para la segunda parte

1.

2.

3.

4.

5.

6.

7.

8.

9.

10.
11.

12.

13.

14.

15.

16.

17.

18.

19.

20. 
Ugarriza 